\title{
On-Line Extraction of SCSI Disk Drive Parameters
}

\author{
Bruce L. Worthington, Gregory R. Ganger, Yale N. Patt, and John Wilkes
}

CSE-TR-323-96

December 19, 1996

Computer Science and Engineering Division

Room 3402, EECS Building

Department of Electrical Engineering and Computer Science

The University of Michigan, Ann Arbor, Michigan 48109-2122 
Copyright December 1996. All rights reserved. 


\title{
On-Line Extraction of SCSI Disk Drive Parameters ${ }^{1}$
}

\author{
Bruce L. Worthington ${ }^{\dagger}$, Gregory R. Ganger ${ }^{\dagger}$, Yale N. Patt ${ }^{\dagger}$, and John Wilkes ${ }^{\ddagger}$ \\ $\dagger$ University of Michigan $\ddagger$ Hewlett-Packard Laboratories \\ 1301 Beal Avenue, EECS Dept. \\ Ann Arbor, MI 48109-2122 \\ 1501 Page Mill Road, MS 1 U13 \\ (313) 936-1602 \\ worthing,ganger,patt@eecs.umich.edu \\ Palo Alto, CA 94304-1126 \\ (415) 857-3568 \\ wilkes@hpl.hp.com
}

December 19,1996

\begin{abstract}
Sophisticated disk scheduling algorithms require accurate and detailed disk drive specifications, including information on mechanical delays, on-board caching and prefetching algorithms, command processing and protocol overheads, and logical-to-physical block mappings. Comprehensive disk models used in storage subsystem design require similar levels of detail. This report describes a suite of general-purpose techniques and algorithms for acquiring the necessary data from SCSI disks via the ANSI-standard interface. The accuracy of the extracted information is demonstrated by comparing the behavior of a configurable disk simulator against actual disk drive activity. Detailed measurements extracted from several SCSI disk drives are provided.
\end{abstract}

\footnotetext{
${ }^{1}$ A shorter version of this report was published in the Proceedings of the 1995 ACM SIGMETRICS Joint International Conference on Measurement and Modeling of Computer Systems, Ottawa, Ontario, May 1995, pp. 146-156 (copyright 1995, ACM Press).
} 


\section{Acknowledgements}

We thank John Fordemwalt, Richie Lary, and Chris Ruemmler for their insights on various disk drive characteristics. We thank Jim Pike of AT\&T/GIS for providing the initial funding for I/O subsystem research at the University of Michigan. We also acknowledge our research groups at the University of Michigan and Hewlett-Packard Laboratories. In particular, Bernard Yeh assisted in our validation efforts. Finally, the University of Michigan research group is very fortunate to have the financial and technical support of several industrial partners, including Hewlett-Packard, AT\&T/GIS, Intel, Motorola, Scientific and Engineering Software, and Digital Equipment Corporation. 


\section{TABLE OF CONTENTS}

1 Introduction 1

2 Modern SCSI disk drives 2

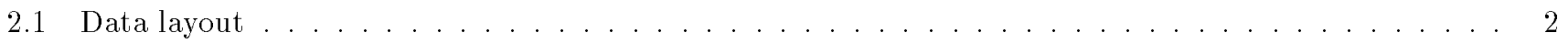

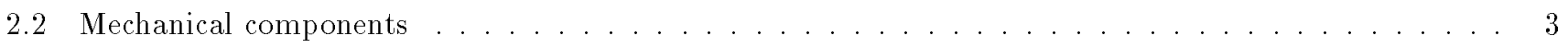

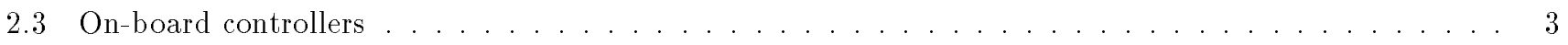

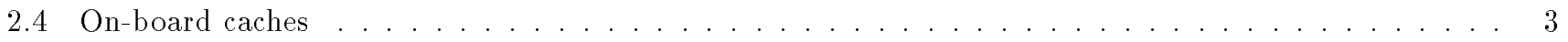

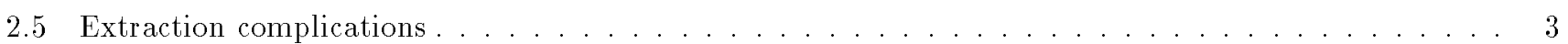

3 Extraction environment 4

4 Interrogative extraction 5

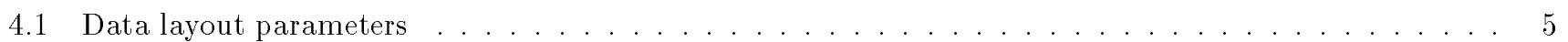

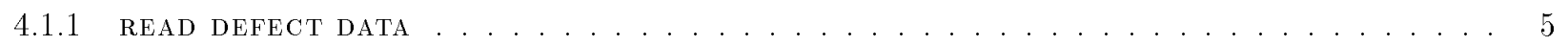

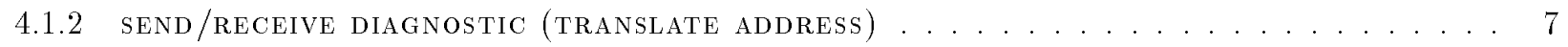

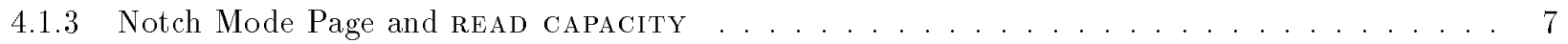

5 Empirical extraction 8

5.1 Minimum time between request completions (MTBRC) ................. . . . . . .

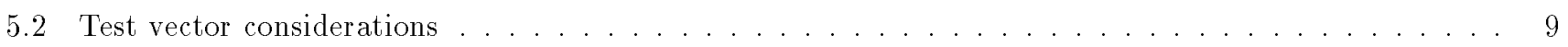

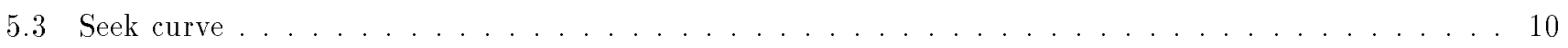

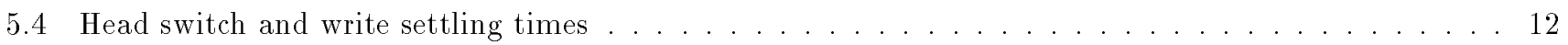

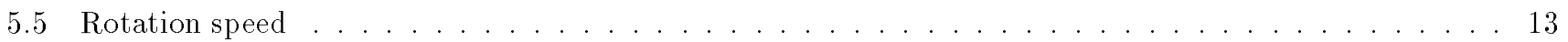

5.6 Command processing and completion overheads . . . . . . . . . . . . . . . . . . . . 13

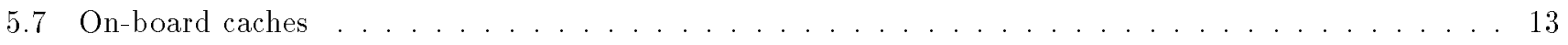

5.7.1 Extraction example: discarding requested sectors after a READ . . . . . . . . . . . . 14

5.7.2 Extraction example: cache segments (number, type, and size) . . . . . . . . . . . . 15

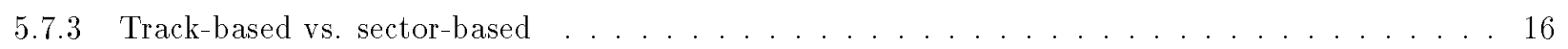

5.7 .4 Read-on-arrival and write-on-arrival . . . . . . . . . . . . . . . . 16

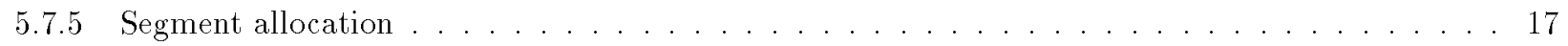

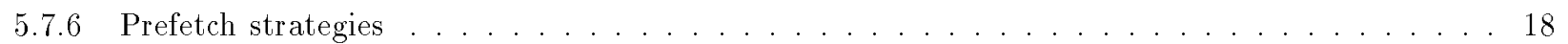




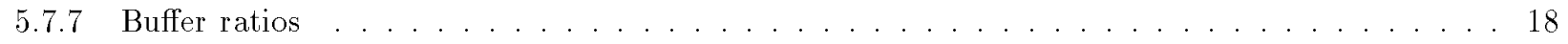

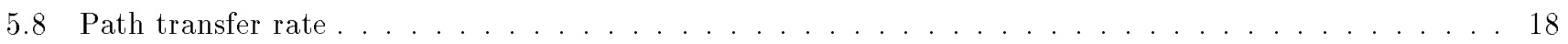

6 Model validation 18

7 Use of extracted data in disk request schedulers 20

8 Conclusions 22

A Extraction algorithms 25

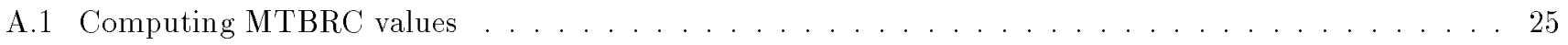

A.2 Command processing and completion overheads . . . . . . . . . . . . . . . . . . . 26

B Extracted parameters for specific disk models 28

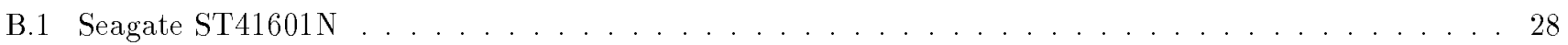

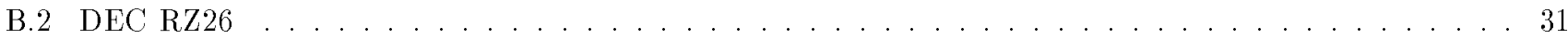

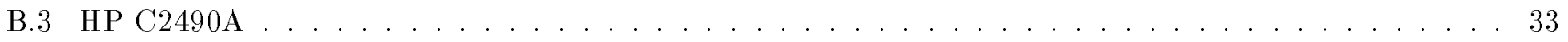

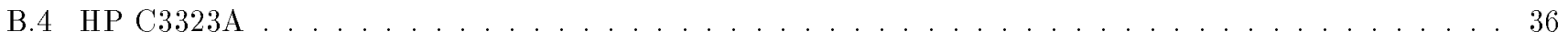




\section{LIST OF FIGURES}

1 Disk drive terminology. . . . . . . . . . . . . . . . . . . . . . . . . . . .

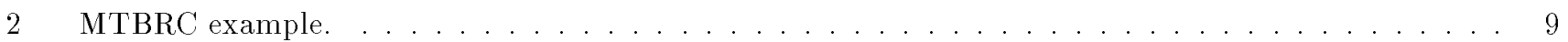

3 Seagate ST41601N: Extracted seek curves. . . . . . . . . . . . . . . . . 11

4 Seagate ST41601N: Service time distributions. . . . . . . . . . . . . . . . . . . 19

5 DEC RZ26: Service time distributions. . . . . . . . . . . . . . . . . . . . . 19

6 HP C2490A: Service time distributions. . . . . . . . . . . . . . . . . . . . . 19

7 HP C3323A: Service time distributions. . . . . . . . . . . . . . . . . . . . 19

8 Seagate ST41601N: Service time densities. . . . . . . . . . . . . . . . . 21

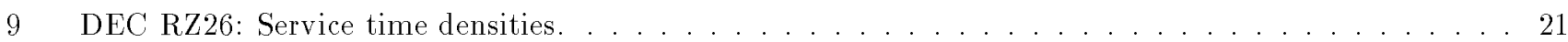

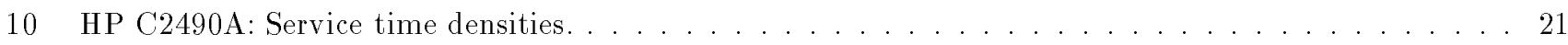

11 HP C3323A: Service time densities. . . . . . . . . . . . . . . . . . . . . . . .

12 Seagate ST41601N: Extracted seek curves. . . . . . . . . . . . . . . . . . . . . . . . .

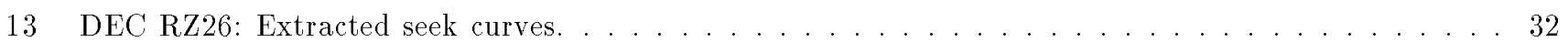

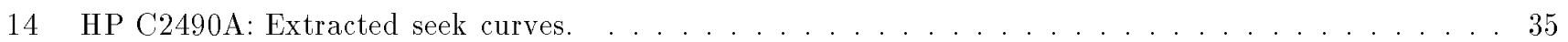

15 HP C3323A: Extracted seek curves. . . . . . . . . . . . . . . . . . . . . . . . . . 


\section{LIST OF TABLES}

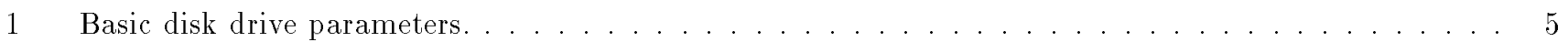

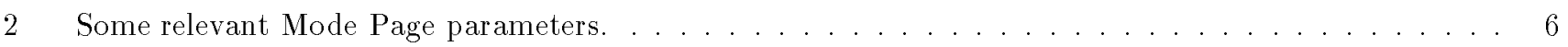

3 Seagate ST41501N: 50th percentile command processing and completion overheads. . . . . . . . 14

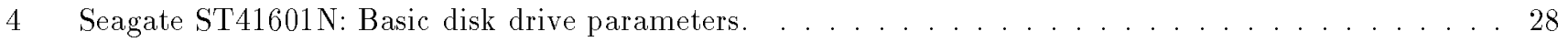

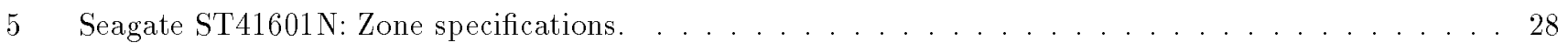

6 Seagate ST41601N: Defect management. . . . . . . . . . . . . . . . . . . 29

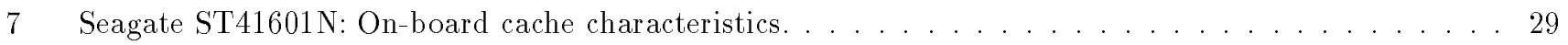

8 Seagate ST41601N: 50th percentile command processing, completion, and mechanical overheads. . . . 29

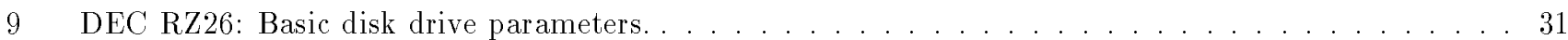

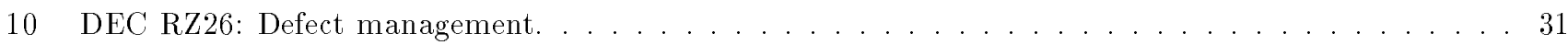

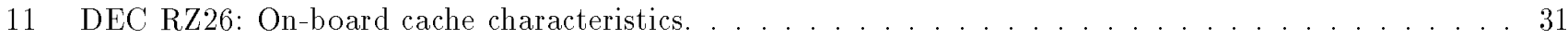

12 DEC RZ26: 50th percentile command processing, completion, and mechanical overheads. . . . . . . 32

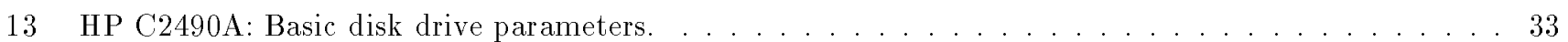

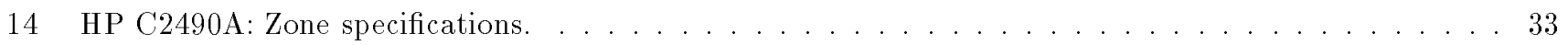

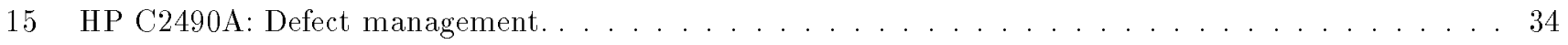

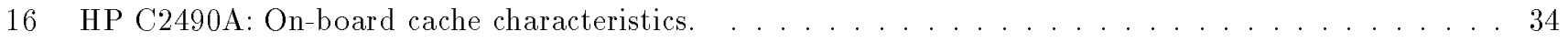

17 HP C2490A: 50th percentile command processing, completion, and mechanical overheads. . . . . . . 34

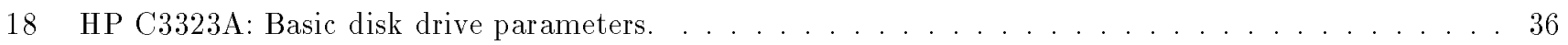

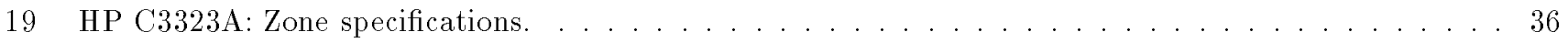

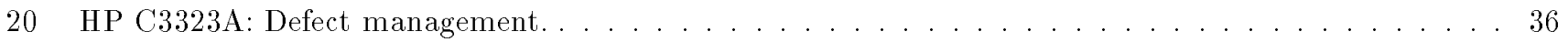

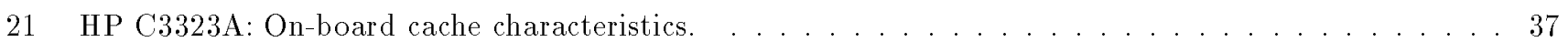

22 HP C3323A: 50th percentile command processing, completion, and mechanical overheads. . . . . . . 37 


\section{Introduction}

The magnetic disk drive remains firmly established as the preferred component for secondary data storage. Given the growing disparity between processor and disk speeds, achieving high system performance requires that disk drives be used intelligently. Previous work has demonstrated that aggressive disk request scheduling algorithms can significantly reduce seek and rotational latency delays [Jacobson91, Seltzer90], and scheduling algorithms that exploit on-board disk caches can further boost performance [Worthington94]. Such algorithms require detailed knowledge of disk characteristics, including mechanical delays, on-board caching and prefetching algorithms, command processing and protocol overheads, and logical-to-physical data block mappings. Accurate disk models utilized in storage subsystem research require similar levels of detail [Ruemmler94].

Information about disk drives can be obtained by reading technical reference manuals, by directly monitoring disk activity at the hardware level, or by talking directly to the drive firmware developers and mechanical engineers. Unfortunately, disk technical manuals are typically incomplete and their accuracy is not guaranteed; direct observation requires expensive signal-tracing equipment and a great deal of time; and it is difficult to reach the correct people at disk drive company XYZ and convince them to release what may be perceived as sensitive data. This report explains how to extract the required information directly from on-line disk drives. In particular, it focuses on disks that conform to the popular Small Computer Systems Interface [SCSI93].

In some cases, standard SCSI commands are used to request parameter values from the disk. These values are typically static in nature, or nearly so. Although such interrogative extraction is convenient, it has two drawbacks: (1) Because the ANSI standard allows a disk drive manufacturer to implement a subset of the full SCSI specification, a given disk drive may not support all interrogative commands. (2) The parameter values returned may be inaccurate or represent nominal figures, such as the mean number of sectors per track on a multi-zone disk.

In cases where interrogation cannot reliably obtain the necessary information, such as for timing-related parameters (e.g., seek times), the host can acquire the values by monitoring disk behavior at the device driver level. Empirical extraction exercises a drive using carefully designed sequences of disk requests, or test vectors. Parameter values are deduced from an analysis of the measured request service times. Much of this report is concerned with the selection of appropriate test vectors that allow quick and accurate determination of parameter values despite the complexity of modern SCSI disk drive controllers.

Depending on the purpose of the extraction, test vectors can be tuned for higher efficiency or greater precision. The default test vectors produce accurate parameter values within minutes. They can be reconfigured to execute in considerably less time (e.g., a few seconds) at the cost of less reliable results. The extraction techniques have been successfully applied to several SCSI disk drives, and accurate disk models have been configured with the measured data. When using the extracted parameter values, detailed disk simulation closely matches real disk behavior.

The remainder of this report is organized as follows. Section 2 contains a brief description of modern SCSI disk drives. For a more detailed discussion, the reader is referred to [Ruemmler94]. Section 3 identifies the system facilities needed for parameter extraction and describes the experimental setup. Sections 4 and 5 describe interrogative and empirical extraction techniques, respectively. Section 6 validates the extraction techniques. Section 7 discusses how disk request schedulers can utilize extracted data. Section 8 contains concluding remarks and suggestions for improving the process of disk characterization. The appendices provide additional detail on specific extraction algorithms and list parameter values extracted from four different SCSI disk drives. 


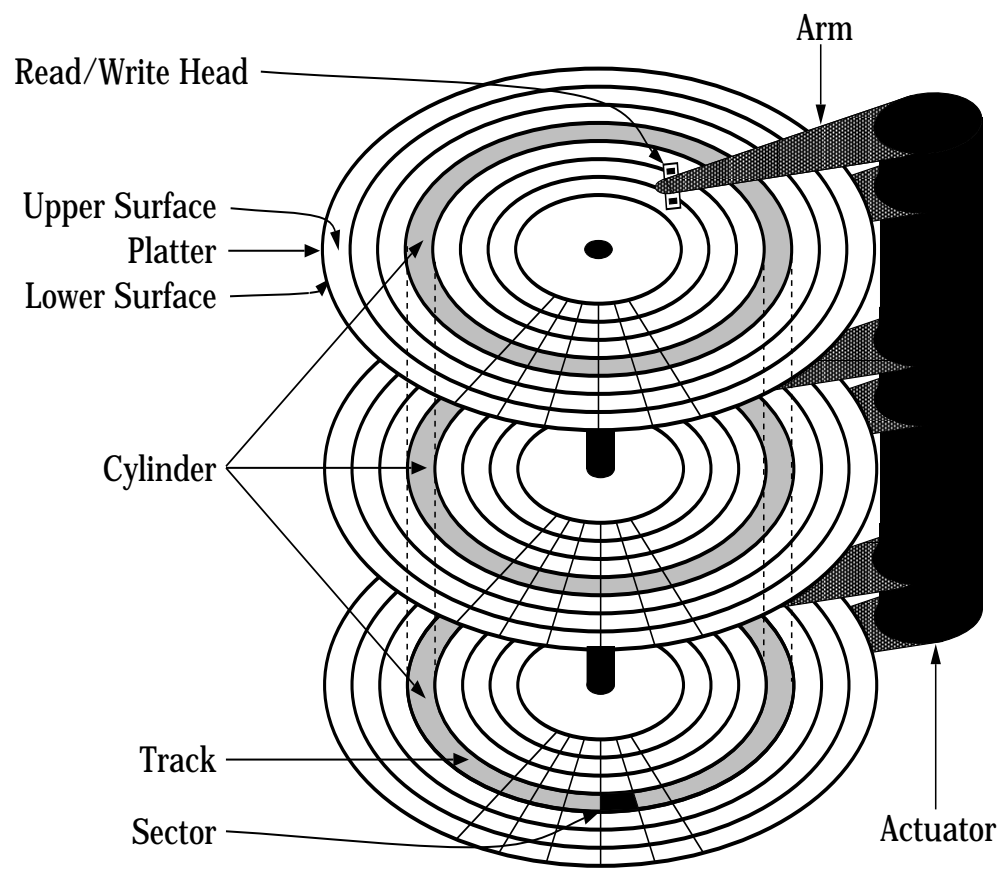

Figure 1: Disk drive terminology.

\section{Modern SCSI disk drives}

\section{$2.1 \quad$ Data layout}

A disk drive consists of a set of rapidly rotating platters (on a common axis) coated on both sides with magnetic media (see figure 1). Data blocks on each surface are written in concentric tracks, which are divided into sectors. A cylinder is a set of tracks (one from each surface) equidistant from the center of the disk. Longer tracks (i.e., those farther out from the axis) can contain more data. To exploit this fact, the set of cylinders may be partitioned into multiple zones or notches. (These two terms are used interchangeably throughout this report.) The number of sectors per track increases from the innermost zone to the outermost zone.

Defects detected during the course of a disk's lifetime require data layout adjustments. Spare regions are reserved to allow for defect slipping and reallocation. Sector (track) slipping takes place during disk formatting: the disk skips over defective sectors (tracks) when initializing the data layout mapping. Each slipped region changes the mapping of subsequent logical blocks. Defect reallocation (a.k.a. remapping or sparing) occurs when defective sectors are discovered during normal disk use. The disk dynamically remaps affected data blocks to spare regions and redirects subsequent accesses accordingly. 


\subsection{Mechanical components}

A disk's read/write heads are held "over" a specific cylinder by a set of disk arms ganged together on a common actuator. Most SCSI disks allow only one active read/write head at any given time. A seek moves the disk arms to a new cylinder. Rotational latency denotes the time spent waiting for the target sector(s) to rotate around to the active read/write head. Switching active heads may require a slight repositioning of the disk arms. The data layout is designed to reduce the performance impact of such head switches on sequential transfers by offsetting the logical-to-physical mapping between logically sequential tracks. The offset, or skew, prevents a request that crosses a track or cylinder boundary from "just missing" the next logical block and waiting almost a full rotation for it to come around again.

\subsection{On-board controllers}

Modern SCSI disks use embedded controllers to decode and service SCSI commands. A host issues a request for disk activity in terms of a starting logical block number (LBN) and a total request size. A disk controller maps the simple linear address space of logical blocks into physical media locations. The details of servicing the request are hidden from the host, offloading most of the management overhead associated with actual data storage from the host (or intermediate I/O controller). As a result, entities outside of the drive typically have little or no knowledge of the exact data layout, the status of the on-board disk cache, and the various overheads and delays associated with servicing a disk request.

Some SCSI commands, such as MODE SENSE, MODE SELECT, and SEND/RECEIVE DIAGNOSTIC, allow access to control and configuration information. If supported by the disk, they can be used during interrogative extraction. Commands normally used to access data, such as READ and wRITE, can be used to empirically extract performance characteristics.

\subsection{On-board caches}

Disk drives originally used on-board memory as a speed-matching buffer between media and bus transfers. Current SCSI drives also use on-board memory as a data cache. On-board caches often contain multiple independent cache lines, or segments, to improve disk performance for workloads containing multiple interleaved sequential data streams. A disk controller can automatically prefetch data into a cache segment to reduce service times for subsequent sequential read requests. Disks with read-on-arrival or write-on-arrival capability can transfer blocks between the on-board cache and the magnetic media in the order they pass under the read/write head rather than in strictly ascending LBN order.

\subsection{Extraction complications}

Disk behavior can appear unpredictable for many reasons, especially when compared against a simple model of disk drive mechanics. Schemes for extracting detailed performance characteristics must cope with the complexities 
of modern disk drives by circumventing certain disk controller optimizations. The extraction techniques discussed in this report handle:

- overlapped disk controller overheads, SCSI bus data transfers, and mechanical delays

- contention for shared resources along the I/O path (e.g., buses and intermediate controllers)

- segmented on-board caches

- simple or aggressive prefetching algorithms

- non-uniform performance characteristics, such as actuator positioning algorithms optimized for very short seek distances

- large, seemingly non-deterministic delays, such as those experienced during thermal recalibration

- minor fluctuations in timing

The existing extraction techniques must be augmented to handle disks with command queuing enabled (i.e., multiple commands queued at the disk). This is left as future work.

\section{Extraction environment}

The described extraction methodology requires a host computer system with direct, low-level SCSI access (bypassing any file system or other translation/caching) and a high-resolution timer to measure the service times of individual disk requests. Some systems (such as HP-UX [Clegg86]) have built-in trace-points that provide the necessary timing capability. In other cases, the extraction techniques may require access to the OS source code, a device driver development environment, or an I/O card that can be directly controlled by an application program (e.g., on a machine running MS/DOS ${ }^{2}$ ).

The extraction experiments described in this report were performed using an NCR 3550 symmetric multiprocessor running an MP-safe version of SVR4 UNIX ${ }^{3}$. Arbitrary SCSI commands were issued directly to specific disk drives using a library of SCSI-related functions provided by NCR. To obtain fine-grained timing data, the device driver was modified to measure service times using a diagnostic counter with a resolution of 840 nanoseconds. Extraneous activity on the host system was minimized to reduce extraction time. Although the algorithms produce valid results in the presence of timing noise (e.g., additional CPU load and bus contention), they take longer to do so.

The extraction techniques were developed and tested using four different disk drives: a Seagate ST41601N [Seagate92, Seagate92a], a DEC RZ26, an HP C2490A [HP93], and an HP C3323A [HP94]. Table 1 lists some basic characteristics of these drives.

\footnotetext{
${ }^{2}$ MS/DOS is a registered trademark of Microsoft Corporation.

${ }^{3} \mathrm{UNIX}$ is a registered trademark of X/Open Corporation.
} 


\begin{tabular}{|c|cccc|}
\hline & $\begin{array}{c}\text { Seagate } \\
\text { ST41601N }\end{array}$ & $\begin{array}{c}\text { DEC } \\
\text { RZ26 }\end{array}$ & $\begin{array}{c}\text { HP } \\
\text { C2490A }\end{array}$ & $\begin{array}{c}\text { HP } \\
\text { C3323A }\end{array}$ \\
\hline Formatted Capacity & $1.37 \mathrm{~GB}$ & $1.03 \mathrm{~GB}$ & $2.13 \mathrm{~GB}$ & $1.05 \mathrm{~GB}$ \\
RPM & 5400 & 5400 & 6400 & 5400 \\
Diameter & $5^{1} /^{\prime \prime}$ & $3^{1} /^{\prime \prime}$ & $3^{1} /^{\prime \prime}$ & $3^{1} /^{\prime \prime}$ \\
Height & $3.25^{\prime \prime}$ & $1.63^{\prime \prime}$ & $1.63^{\prime \prime}$ & $1.00^{\prime \prime}$ \\
Data Surfaces & 17 & 14 & 18 & 7 \\
Cylinders & 2098 & 2570 & 2582 & 2910 \\
Zones & 14 & 1 & 11 & 8 \\
Sectors/Track & $61-85$ & 58 & $68-108$ & $72-120$ \\
\hline
\end{tabular}

Table 1: Basic disk drive parameters.

\section{Interrogative extraction}

SCSI disk drives can supply a number of parameter values upon request. Most disks implement both ANSIstandard and vendor-specific methods for requesting configuration information. Unless otherwise noted, the extraction techniques use only ANSI-standard features supported by all of the modern SCSI disks in the authors' library of disk specifications from DEC, Fujitsu, Hewlett-Packard, Maxtor, Micropolis, Quantum, and Seagate.

SCSI Mode Pages allow on-line access to various disk drive configuration parameters. Table 2 lists some relevant Mode Page parameters. ${ }^{4}$ Each Mode Page holds a particular type of information, such as Caching, Rigid Disk Drive Geometry, or Read-Write Error Recovery. The MODE SENsE command returns the contents of a specific Mode Page. The MODE SELECT command updates the contents of a specific Mode Page.

Unfortunately, Mode Page information may be misleading. In some cases, the standard SCSI Mode Pages cannot adequately reflect the behavior of the disk controller. In other cases, the manufacturer may have decided that a nominal value would suffice. As a result, empirical extraction methods (see section 5 ) should be used to verify data acquired via interrogative extraction.

\subsection{Data layout parameters}

An accurate logical-to-physical mapping of individual data blocks is vital for disk scheduling and modeling efforts. The described extraction methodology relies heavily on the READ DEFECT DATA command and the TRANSLATE ADDRESS form of the SEND/RECEIVE DIAGNOSTIC commands.

\subsubsection{READ DEFECT DATA}

Previous work has shown that scheduling algorithms and disk models can ignore reallocated defects in most cases [Worthington94]. Slipped defects, on the other hand, can cause major data layout perturbations, especially in

\footnotetext{
${ }^{4}$ In this report, hexadecimal values are prefixed with "0x", byte offsets are prefixed with "B", and bit offsets are prefixed with "b". Byte and bit offsets start at zero.
} 


\begin{tabular}{|c|c|}
\hline Mode Page \& Parameter & Location \\
\hline \multicolumn{2}{|l|}{ "Disconnect-Reconnect (0x02) } \\
\hline Buffer Full Ratio & B2 \\
\hline Buffer Empty Ratio & B3 \\
\hline Bus Inactivity Limit & B4-B5 \\
\hline Disconnect Time Limit & B6-B7 \\
\hline Connect Time Limit & B8-B9 \\
\hline Maximum Burst Size & B10-B11 \\
\hline Data Transfer Disconnect Control & $\mathrm{B} 12 \mathrm{~b} 0-\mathrm{b} 1$ \\
\hline \multicolumn{2}{|l|}{ Format Device (0x03) } \\
\hline Tracks Per Zone $e^{a d}$ & B2-B3 \\
\hline Alternate Sectors Per Zone ${ }^{a d}$ & B4-B5 \\
\hline Alternate Tracks Per Zone ${ }^{a d}$ & B6-B7 \\
\hline Alternate Tracks Per Logical Unit ${ }^{a}$ & $\mathrm{~B} 8-\mathrm{B} 9$ \\
\hline Sectors Per Track ${ }^{a}$ & B10-B11 \\
\hline Data Bytes Per Physical Sector ${ }^{a}$ & $\mathrm{~B} 12-\mathrm{B} 13$ \\
\hline Interleave $^{a b}$ & B14-B15 \\
\hline Track Skew Factor ${ }^{a}$ & $\mathrm{~B} 16-\mathrm{B} 17$ \\
\hline Cylinder Skew Factor $^{a}$ & B18-B19 \\
\hline Surface Mode ${ }^{c}$ & $\mathrm{~B} 20 \mathrm{~b} 4$ \\
\hline \multicolumn{2}{|l|}{ Rigid Disk Geometry (0x04) } \\
\hline Number of Heads & B5 \\
\hline Medium Rotation Rate & B20-B21 \\
\hline \multicolumn{2}{|l|}{ Caching (0x08) } \\
\hline Read Cache Disable & $\mathrm{B} 2 \mathrm{~b} 0$ \\
\hline Multiplication Factor & $\mathrm{B} 2 \mathrm{~b} 1$ \\
\hline Write Cache Enable ${ }^{c}$ & $\mathrm{~B} 2 \mathrm{~b} 2$ \\
\hline Disable Prefetch Transfer Length & B4-B5 \\
\hline Minimum Prefetch & B6-B7 \\
\hline Maximum Prefetch & B8-B9 \\
\hline Maximum Prefetch Ceiling & $\mathrm{B} 10-\mathrm{B} 11$ \\
\hline \multicolumn{2}{|l|}{ Notch $(0 \times 0 C)$} \\
\hline Logical Or Physical Notch ${ }^{a}$ & $\mathrm{~B} 2 \mathrm{~b} 6$ \\
\hline Maximum Number of Notches & B4-B5 \\
\hline Active Notch & $\mathrm{B} 6-\mathrm{B} 7$ \\
\hline Starting Boundary ${ }^{a}$ & B8-B11 \\
\hline Ending Boundary $^{a}$ & $\mathrm{~B} 12-\mathrm{B} 15$ \\
\hline
\end{tabular}

a Active Notch must be set via Mode Select before requesting per-notch values.

${ }^{b}$ Must be zero or one during extraction.

${ }^{c}$ Must be zero during extraction.

${ }^{d}$ For this parameter, "Zone" refers to a region over which spare sectors are allocated. In other contexts, "Zone" is a common synonym for "Notch."

Table 2: Some relevant Mode Page parameters. 
the case of track-based slipping. As reallocated defects may be converted into slipped defects during disk format, data layout extraction should be repeated after reformatting. The READ DEFECT DATA command requests a list of defective sectors (or tracks) from a disk in cylinder/head/sector (or cylinder/head) format. To obtain a complete list of all defects detected during and after manufacture, both the Primary Defect List Bit (B2b4) and Grown Defect List Bit (B2b3) must be set in the READ DEFECT DATA command descriptor block.

\subsubsection{SEND/RECEIVE DIAGNOSTIC (TRANSLATE ADDRESS)}

The tRanslate ADDRESS form of the SEND Diagnostic command requests the exact logical-to-physical mapping for a single logical block. The corresponding physical media location (cylinder/head/sector) is returned by a subsequent RECEIVE DIAGNOSTIC command. Obtaining a full logical-to-physical mapping by iterating through every logical block number can take several hours. A variety of shortcuts are used to reduce the number of SEND/RECEIVE DIAGNOSTIC command pairs required to obtain a complete mapping. The extraction algorithms first determine the boundaries, sectors per track, track skew, and cylinder skew for each zone, or verify this information if it was obtained from the Mode Pages. The current mapping is then requested for the "expected" first and last logical block of each cylinder. Alternately, a faster algorithm might examine only a fraction of the total set of cylinders. Mismatches between actual and expected values indicate the existence of slipped defects or spare regions, which can then be pinpointed with additional SEND/RECEIVE DIAGNOSTIC command pairs in a binary search pattern.

\subsubsection{Notch Mode Page and READ CAPACITY}

Unfortunately, there is at least one major disk manufacturer, Quantum, that does not always provide TRANSLATE ADDRESS functionality. For drives lacking this capability, an alternate methodology for extracting data layout information is required. In the specific case of Quantum disk drives, many of the necessary parameter values can be obtained using the Notch Mode Page and the READ CAPACITY command.

When a disk supports both the Notch Mode Page and the Format Device Mode Page (see table 2), it is possible to obtain most of the necessary mapping information using the Mode Select command. The combination of parameter values from these two pages specifies the data layout and size of each notch. The READ CAPACITY command can be used to determine the logical boundaries of each cylinder, provided that the implementation of the command allows for the retrieval of individual cylinder capacities. This information can be used to identify deviations from the per-notch Mode Page parameter values. Additional empirical extraction is still necessary to validate the Mode Page parameter values as well as to obtain data layout information that cannot be interrogatively extracted without TRANSLATE ADDRESS functionality. For example, the skew between the last sector of a given notch and the first sector of the next notch is not specified by any Mode Page field. Also, not all defect-handling schemes can be adequately represented using the fields of the Format Device Mode Page.

Complete details of the alternate data layout extraction methodology using the Notch Mode Page and READ CAPACITY command are not included in this report. 


\section{$5 \quad$ Empirical extraction}

Although some disk parameters and characteristics can be obtained via interrogation, many must be deduced by observing dynamic disk behavior. This section describes various empirical extraction algorithms and their use in measuring disk performance characteristics. In particular, it contains descriptions of a general empirical extraction methodology, algorithms for extracting mechanical parameter values, algorithms for approximating command processing and completion overheads, techniques for characterizing cache behavior, and a simple algorithm for computing an overall I/O path transfer rate.

For modeling purposes, mean or 50th percentile parameter values are often sufficient. For other purposes, (probabilistic) upper bound values may be necessary. For example, a disk request scheduler configured with mean values will frequently underpredict command processing overheads and mechanical positioning times, occasionally resulting in excessive rotational delays. It may be advantageous to use conservative parameter values instead, limiting the opportunity for unacceptable delays. For this reason, maximum or 95th percentile values are also extracted.

\subsection{Minimum time between request completions (MTBRC)}

Many of the empirical extraction techniques rely on measuring the minimum time between request completions for specific pairs of request types. $M T B R C(\mathrm{X}, \mathrm{Y})$ denotes the minimum time between the completions of a request of type $\mathrm{X}$ and a request of type $\mathrm{Y}$. For example, MTBRC(1-sector write, 1-sector read on an adjacent cylinder) refers to the minimum time between request completions for a 1-sector write request followed by a 1-sector read request requiring a 1-cylinder seek.

The determination of an accurate MTBRC value is an iterative process, wherein the inter-request distance between each pair of requests (i.e., between each type $\mathrm{X}$ and type $\mathrm{Y}$ request) is varied until the minimum time between request completions is observed. The inter-request distance is the rotational distance between the physical starting locations of each MTBRC request pair, measured along the direction of rotation. When the X requests are for small amounts of data and the $\mathrm{Y}$ requests do not incur significant seek times, inter-request distances are only a fraction of a full rotation. So, for example, MTBRC(1-sector write, 1-sector read on the same track) might have a corresponding inter-request distance of 25 sectors, given a fixed number of sectors per track. Assuming sectors 100 and 125 are on the same track, the minimum time between request completions would be observed for a 1-sector write to sector 100 followed by a 1-sector read from sector 125. If the number of sectors per track varies across the disk, then the interrequest distance can be measured as the angle between the first sectors of the request pairs, or as the time necessary for the disk to rotate through this angle. Appendix A.1 provides a detailed algorithm for extracting MTBRC values.

To determine the MTBRC for a given request pair specification, the iterative MTBRC algorithm finds the interrequest distance for which the second request of each pair incurs essentially zero rotational latency. Any rotational latency that is not in parallel with command processing overheads or mechanical delays must be less than the time necessary to rotate past one sector of data. Otherwise, a shorter inter-request distance would result in a lower time between request completions.

The algorithm also computes the mean host delay. The host delay is the time between the completion of the first request of a request pair and the initiation of the second request. This delay is due solely to processing time at the host (e.g., executing device driver code, configuring intermediate controllers, and copying data from kernel memory 


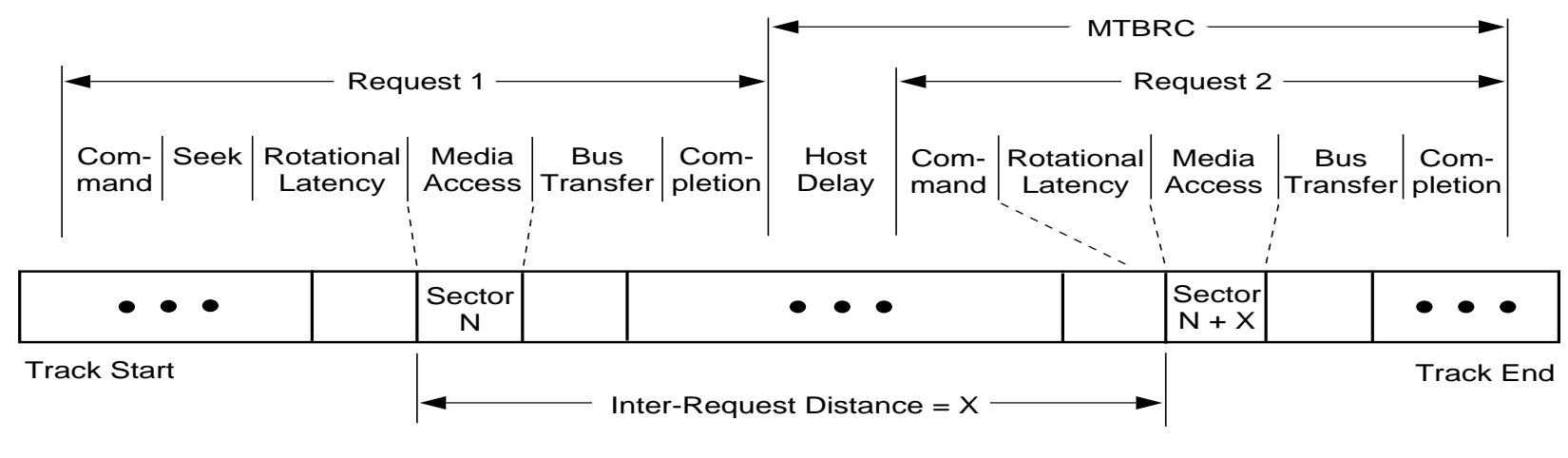

TIME

Figure 2: MTBRC example. The time-line shows the service time components for an example MTBRC(1-sector READ, 1-sector READ on same track) request pair. The track accessed by the two requests is also shown, but as a straight line rather than as a circle. Timestamps are taken at the initiation and completion of each request, providing the host delay and the MTBRC timings. The inter-request distance is the difference between the starting locations of the two requests.

to user space). Mean host delays can be subtracted from MTBRC values to remove variations in host processing times. Figure 2 shows the service time components for an example MTBRC request pair.

In order to determine a value for a specific disk parameter, MTBRC values are collected for two or more sets of selected request type pairs. Each MTBRC value is the sum of several discrete service time components. Specific component values are isolated via simple algebraic techniques. In section 5.4, for example, the effective head switch time is computed from $\mathrm{MTBRC}_{1}$ (1-sector write, 1-sector read on the same track) and $\mathrm{MTBRC}_{2}(1$-sector write, 1 -sector read on a different track of the same cylinder). The corresponding equations are:

$$
\begin{aligned}
& M T B R C_{1}=\text { HostDelay } 1+\text { Command }+ \text { Media }+ \text { Bus }+ \text { Completion } \\
& M T B R C_{2}=\text { HostDelay }+ \text { Command }+ \text { HeadSwitch }+ \text { Media }+ \text { Bus }+ \text { Completion }
\end{aligned}
$$

Via substitution and reorganization:

$$
\text { HeadSwitch }=\left(M T B R C_{2}-\text { HostDelay }_{2}\right)-\left(M T B R C_{1}-\text { HostDelay }\right)
$$

\subsection{Test vector considerations}

Empirical extraction test vectors range from tens to thousands of requests in length. In many cases, a large fraction of the requests are intended to defeat controller optimizations and eccentricities. Test vectors must be carefully designed to prevent unexpected mechanical delays and/or interference from the on-board cache, even when the cache is only functioning as a speed-matching buffer:

- As some disks must "warm-up" after they have been idle for some period of time, each test vector is prefixed by 20-50 random disk requests whose service time measurements are discarded. Without this precaution, the first 10-20 requests of a test vector can take up to ten times longer than normal to complete. 
- Any on-board write-back (a.k.a. fast-write) cache activity should be disabled during extraction by setting the Write Cache Enable Bit to zero (see table 2).

- When it is necessary to force cache misses for READ requests, the cache must be appropriately initialized. If the cache uses a Least Recently Used (LRU) segment replacement algorithm, for example, initialization involves issuing a number of physically scattered READs equal to the maximum number of cache segments.

- As read requests often invoke automatic prefetching activity that may result in unwanted switching between tracks (or cylinders), test vectors should use WRITE requests or choose initial MTBRC READ targets from the lowest LBNs on a track. The latter option relies on host delay values being short enough and/or rotation speeds being slow enough to allow any positioning activity for the second request to be initiated before the logical end of the current track is reached.

- The overlap between media and bus transfers can be eliminated by using 1-sector requests or by setting the Buffer Ratio fields (see table 2) to values that minimize data transfer pipelining, provided that the disk controller obeys these values. Specifically, a large Buffer Full Ratio will cause the disk controller to wait for all READ data to be cached before reconnecting to the bus. It is more difficult to prevent overlap between WRITE data transfer and media access. If multi-sector wRITE requests are required, they should be designed to incur long mechanical delays, thereby increasing the probability that all data transfer over the bus is completed prior to any media access.

\subsection{Seek curve}

A seek curve graph displays seek time as a function of seek distance. The seek time between two cylinders is a complex function of the position of the cylinders (i.e., their distance from the spindle), the direction of the seek (inward or outward), various environmental factors (e.g., vibration and thermal variation), the type of servo information (dedicated, embedded, or hybrid), the mass and flexibility of the head-arm assembly, the current available

to the actuator, etc. A seek curve attempts to reduce this complexity into a single mapping of seek distances to seek times.

Different algorithms may be used for extracting seek curves depending on whether the end goal is accurate disk modeling or high-performance disk request scheduling. A disk model should use a seek curve that reflects mean seek times for each seek distance. A mean seek curve may be inexact for any given request, but it should result in a good overall emulation of seek behavior. A disk scheduler, on the other hand, may be better served by a more conservative seek curve containing near-maximum seek times.

The following algorithm extracts two seek curves. It requires an accurate logical-to-physical map (see section 4.1) and the use of the SEEk command. The first seek curve consists of mean seek times for specific seek distances. The second curve, denoted as the "maximum" seek curve, gives an approximate upper bound on seek times for the same set of seek distances.

1. For each seek time to be extracted, select 5 starting points evenly spaced across the physical cylinders. For longer seek distances, the number of starting points may be decreased. Full stroke seeks, for example, must necessarily start on either the innermost or outermost cylinder. From each starting point, perform 10 inward and 10 outward SEEKs of the appropriate distance. While most of the measured service times should be roughly equivalent, there will often be anomalous values caused by non-deterministic disk activity (e.g., thermal recalibration or mis-reads), periodic host activity (e.g., clock interrupts), or unexpected contention for other shared resources (e.g., SCSI 


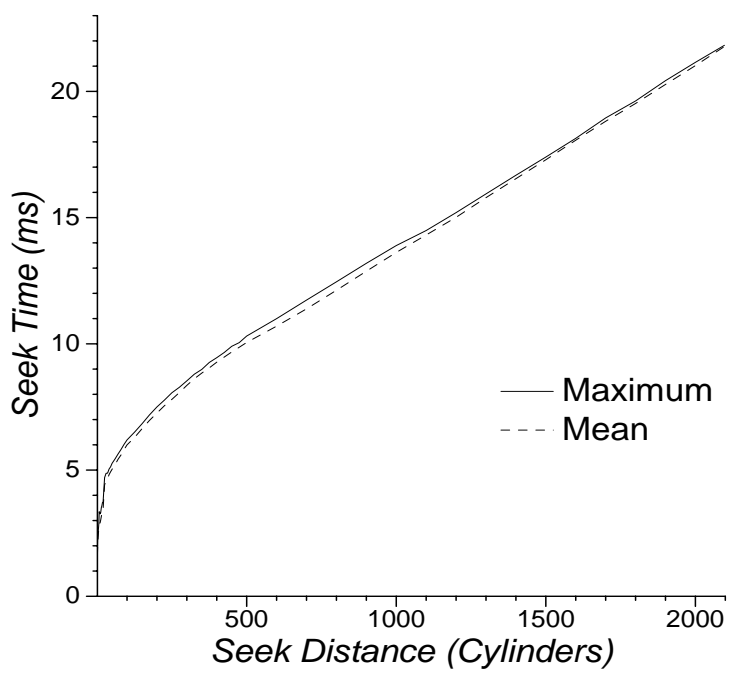

(a) Full seek curve

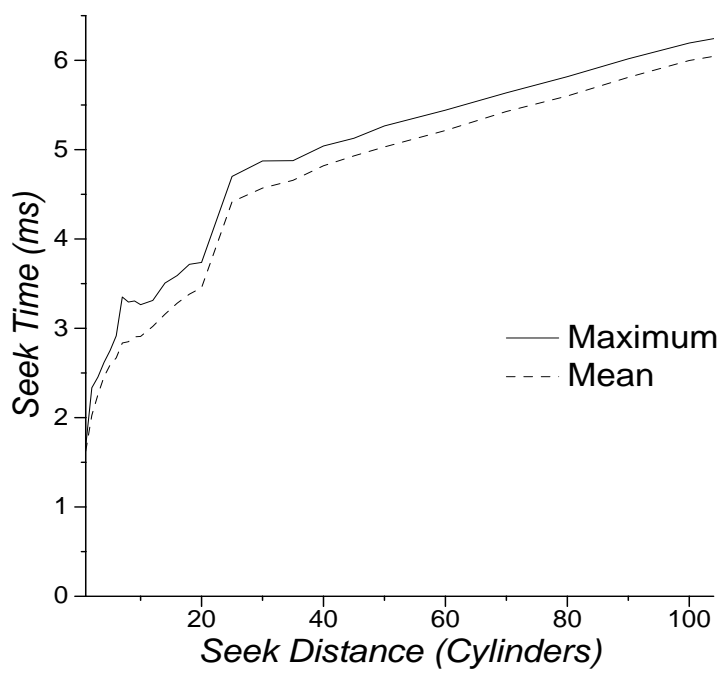

(b) Expanded view of short seeks

Figure 3: Seagate ST41601N: Extracted seek curves.

bus traffic involving other disks). Excessive service times may be omitted if such activities are to be ignored by the disk model or scheduler. For the seek curves given in this report, service times deviating from the median value by more than $10 \%$ were discarded.

2. Average the 5 sets of measured service times to obtain a single mean service time value for each of the 5 starting points.

3. For the mean seek curve, average the 5 mean service time values. For the maximum seek curve, take the maximum of the 5 values. Thus the maximum seek curve extracted by this algorithm reflects mean seek times for regions of the disk which generally experience the longest seek times. If tighter guarantees on response times are desired, other algorithms might be more appropriate. For example, 95th percentile values could be computed for each of the 5 sets of measured service times, and the maximum seek curve could be comprised of the maximums of the 95th percentile values. Alternately, partial seek curves could be extracted for different regions of the disk.

4. After the mean and maximum service times have been determined for each seek distance, the portion of the service time due to actuator movement must be isolated. A corrective value is computed from MTBRC(1-sector write, 1-sector read on the same track) and MTBRC(1-sector write, 1-sector read on an adjacent cylinder). The difference between these two values effectively represents the time necessary to mechanically seek across one cylinder. The difference between this value and the extracted 1-cylinder SEEK service time represents the nonmechanical overheads associated with processing a SEEK request. This processing delay should be subtracted from each extracted service time. The resulting values comprise the extracted mean and maximum seek curves.

Figure 3 shows the extracted seek curves for a Seagate ST41601N. As the seek distance increases, seek curves become linear. In fact, most seek times for larger seek distances can be computed using linear interpolation, thereby reducing the number of actual measurements necessary. The seek curves in figure 3 contain seek times for every seek 
distance between 1 and 10 cylinders, every 2nd distance up to 20 cylinders, every 5 th distance up to 50 cylinders, every 10 th distance up to 100 cylinders, every 25 th distance up to 500 cylinders, and every 100 th seek distance beyond 500 cylinders. Using this method, the extraction process takes less than two minutes for each of the four disks tested. By reducing the number of starting points, repetitions, and/or measured seek distances, the extraction time can be reduced with some loss of accuracy.

\subsection{Head switch and write settling times}

The time necessary to switch read/write heads depends primarily on the type of servo information utilized by the disk drive (dedicated, embedded, or hybrid) and various environmental factors, such as vibration and thermal variation. For the current generation of disk drives, any seek activity will typically subsume the head switch time. WRITE requests incurring head switches or seeks may require additional settling time to more closely align the read/write heads. Data can be read from the media successfully even when the read/write heads are slightly misaligned, but data must be written as close to the middle of a track as possible in order to prevent corruption of adjacent tracks of data. So, the time necessary to position the actuator and activate the appropriate read/write head is calculated as follows:

$$
\begin{aligned}
& \text { ActuatorPrep } p_{S e e k}=\text { Seek }(+ \text { WriteSettle if WRITE }) \\
& \text { ActuatorPrep } p_{\text {NoSeek }}=\text { HeadSwitch }(+ \text { WriteSettle if WRITE })
\end{aligned}
$$

Since head switch and write settling times cannot be measured directly, the extraction algorithms compute effective values that are sufficient for disk modeling and request scheduling purposes.

The effective head switch time is the portion of the head switch time that is not overlapped with command processing or data transfer. It can be computed from $\mathrm{MTBRC}_{1}$ (1-sector write, 1-sector read on the same track) and $\mathrm{MTBRC}_{2}$ (1-sector write, 1-sector read on a different track of the same cylinder), as described in section 5.1:

$$
\text { HeadSwitch }=\left(M T B R C_{2}-\text { Host Delay }_{2}\right)-\left(M T B R C_{1}-\text { HostDelay }\right)
$$

The effective write settling time is the portion of the write settling time that is not overlapped with command processing or data transfer. It can be computed from the effective head switch time, $\mathrm{MTBRC}_{1}$ (1-sector write, 1 -sector write on same track), and $\mathrm{MTBRC}_{2}$ (1-sector write, 1-sector write on a different track of the same cylinder):

$$
\text { WriteSettle }=\left(\left(M T B R C_{2}-\text { HostDelay }_{2}\right)-\left(M T B R C_{1}-\text { HostDelay } 1\right)\right)-\text { HeadSwitch }
$$

The difference between the two MTBRC values (after accounting for any difference in mean host delays) is the sum of the effective head switch and effective write settling times. The effective write settling time is isolated by subtracting out the effective head switch time. Negative effective write settling times were computed for some of the disks tested, indicating that additional overlap occurs between head switching/write settling and command processing/bus transfer for WRITEs. A negative effective write settling time poses no problem as long as the overall actuator positioning and head switch delay (ActuatorPrep) is positive. For disks using only dedicated servo information, such as the Seagate ST41601N, the extracted effective head switch and same-cylinder write settling times may be negligible. But write settling is still a factor for requests that incur seek activity. An effective "seek-only" write settling time can 
be computed for such disks by modifying the above algorithm to use $\mathrm{MTBRC}_{2}$ (1-sector write, 1-sector write on an adjacent cylinder).

Track skew can be used as a high-probability upper bound on the combined head switch and write settling delays. Disk manufacturers set track skew values to minimize the interruption in media access incurred when sequential transfers cross track boundaries.

\subsection{Rotation speed}

Rotation speed can be determined empirically by performing a series of 1-sector wRITEs to the same location and calculating the mean time between request completions. A small number of repetitions (e.g., 32) should be sufficient to obtain an accurate value. The mean time between completions is equal to the time per rotation, which is the reciprocal of rotation speed. Extracted rotation speeds for the four disks tested are within manufacturer-specified tolerances.

\subsection{Command processing and completion overheads}

Given a "black-box" extraction methodology, it is difficult to measure certain parameters directly. Command processing, completion, and other overheads can only be measured as combined quantities unless the extraction process has access to timing information for when actual data transfers begin and end. Command processing and completion overheads can depend on the type of command (READ or WRITE), the state of the cache (hit or miss), and the immediately previous request's characteristics [Worthington94]. Overhead values can be approximated by solving multiple linear equations obtained from MTBRC extractions for the various unknown values. For disk models, the level of detail desired determines the number of different MTBRC extractions necessary. For disk schedulers, combined quantities can be used directly (see section 7 ).

To configure a disk simulator for validation purposes (see section 6), ten equations containing eight unknowns (six command processing overheads and two completion overheads) were solved for each of the four disks tested. When inconsistent equations suggested conflicting values for an overhead, the mean of the conflicting values was used. It was also necessary to reduce the number of unknowns by arbitrarily setting one unknown to zero (see section A.2).

Table 3 shows extracted and published overhead values for the Seagate disk drive. Note the significant variation between processing overhead values for different types of commands. The difference between extracted and published values is due in part to the fact that extracted values include some host delay, SCSI protocol overhead, and concurrent mechanical activity.

\subsection{On-board caches}

The standard SCSI specification provides for a wide range of cache-management policies, and manufacturers are free to extend this range. This section discusses a methodology for characterizing on-board disk caches. The methodology consists of a series of experiments (i.e., test vectors) that prove or disprove specific hypotheses about cache behavior. Boolean parameter values (e.g., can a READ hit on cached data from a previous wRITE?) typically require only one test vector. Tests for cache parameters that can take on several values (e.g., the number of cache 


\begin{tabular}{|l|c|}
\hline \multicolumn{1}{|c|}{ Overhead } & Value \\
\hline \hline Read Hit After Read & $0.895 \mathrm{~ms}$ \\
Read Hit After Write & $0.497 \mathrm{~ms}$ \\
Read Miss After Read & $1.442 \mathrm{~ms}$ \\
Read Miss After Write & $1.442 \mathrm{~ms}$ \\
Write After Read & $2.006 \mathrm{~ms}$ \\
Write After Write & $1.473 \mathrm{~ms}$ \\
\hline Read Completion & $0.239 \mathrm{~ms}$ \\
Write Completion & $0.000 \mathrm{~ms}$ \\
\hline Published Per-Command & $0.700 \mathrm{~ms}$ \\
\hline
\end{tabular}

Table 3: Seagate ST41501N: 50th percentile command processing and completion overheads.

segments) use feedback from each hypothesis to help select the next hypothesis and test vector. Designing a complete set of hypotheses is difficult, if not impossible; the on-board cache can only be characterized to the extent of the extraction algorithms' knowledge of possible cache characteristics. The current suite of extraction algorithms successfully characterized the cache behavior of the four disks tested.

Algorithms that extract cache parameter values generally contain three phases: (1) A hypothesis about cache behavior is made. (2) The cache is loaded with data through the issuing of one or more specific disk requests. (3) The contents of the cache are inspected by issuing a single READ request and determining whether or not the request hit in the on-board cache. The requests issued in phases two and three comprise a test vector.

The following sections give several examples to demonstrate this extraction technique and discuss the types of parameters that characterize an on-board disk cache.

\subsubsection{Extraction example: discarding requested sectors after a READ}

Some on-board cache management algorithms give precedence to prefetched data over requested data. Such algorithms assume that subsequent requests are more likely to access sequential data than previously requested data. This optimization can be implemented in several different ways. Prefetching activity may "push" requested data out of a cache segment once the segment is full, thereby allowing additional sectors to be prefetched. Alternately, requested sectors can be discarded from the cache as soon as they are transferred to the host, leaving the entire segment available for prefetching activity. The following algorithm tests for the latter option.

Choosing the hypothesis

1. Assume that sectors requested by a READ are discarded from the cache before the request is considered complete. Loading the cache

2. Perform a 1-sector READ of a randomly-chosen logical block. 
Testing the hypothesis

3. Perform a 1-sector READ of the same logical block. The request should incur almost a full rotation of latency, since the requested sector should no longer exist in the on-board cache. A request response time in excess of $3 / 4$ of a rotation should be sufficient indication that a cache miss occurred, assuming that completion and cleanup, host, and protocol delays take up less time than $1 / 4$ of a rotation.

If the second request completes in less than $3 / 4$ of a rotation, a cache hit has occurred, the test has failed, and it can be assumed that the target sectors of a READ request remain in the on-board cache after the request has completed.

\subsubsection{Extraction example: cache segments (number, type, and size)}

Although modern SCSI disk drives typically contain Mode Page data regarding the characteristics of on-board cache segments, such information is not required or well-specified by the ANSI standard. For this reason, empirical extraction techniques are necessary to obtain or validate cache segment configuration and management data.

The following algorithm determines the maximum number of cache segments available to READs:

Choosing the initial hypothesis

1. Set N, the hypothesized number of READ segments, to a large value (e.g., 32).

Loading the cache

2. Perform 1-sector READs of the first logical blocks of the first N-1 data cylinders. By only accessing blocks that are at the logical "beginnings" of their respective cylinders, the possibility of unwanted head switchs and seeks caused by automated prefetching activity is minimized (see section 5.2).

3. Perform a 1-sector READ of the first logical block of the last data cylinder. This will cause the next request to incur a full-stroke seek.

Testing the hypothesis

4. Perform a 1-sector READ of the first logical block of the first data cylinder. A cache hit should occur if the number of cache segments is $\mathrm{N}$ or greater, assuming a segment replacement scheme such as LRU or FIFO. A request response time of less than $3 / 4$ of a rotation should be sufficient indication that a cache hit occurred, assuming that a full-stroke seek takes more than $3 / 4$ of a rotation.

Choosing the next hypothesis

5. If step 4 results in a cache miss, the test has failed. Decrement $\mathrm{N}$ and return to step 2. If step 4 results in a cache hit, the test has succeeded and the cache has at least $\mathrm{N}$ READ segments. If $\mathrm{N}$ is the largest hypothesized value that has been tested, increment $\mathrm{N}$ and return to step 2. Otherwise, there are exactly N READ segments. To increase the algorithm efficiency, a standard binary search can be employed.

This algorithm is designed for caches with multiple segments. The existence of a single cache segment can be detected by issuing two 1-sector READs for the same location and determining whether or not the second READ hits in the cache. If the second request misses, it will incur almost a full rotation of latency. A request response time in 
excess of $3 / 4$ of a rotation should be sufficient indication that a cache miss occurred, assuming that completion and cleanup, host, and protocol delays take up less time than $1 / 4$ of a rotation. If no segments are detected, the first algorithm should be repeated with step 4 modified to read the second logical block from the first data cylinder. This will handle disks that discard requested data after completing a READ (see section 5.7.1). It assumes that each of the requests result in a small amount of automated prefetching activity before the servicing of the next request.

Some on-board caches have dedicated segments for use by wRITEs, while others allow all segments to be used by READs or writes. Additional test vectors can determine which scheme has been implemented. For example, steps 2 and 3 can be modified to include one or more wRITEs. If the extracted number of cache segments increases, one or more cache segments are dedicated to WRITEs. If the extracted number of segments remains unchanged, some or all of the segments are usable by either READs or WRITEs.

Extracting the size of the cache segments can be quite simple or very difficult depending on the prefetching behavior of the disk. The basic extraction technique computes the segment size by filling a cache segment with data (e.g., via a large READ) and then determining the boundaries of the segment by detecting READ hits (or misses) against the cached data. Sufficient time is allowed for all prefetching activity to cease before probing the segment boundaries with 1-sector READ requests. Of course, the cache segment must be re-initialized after each "probe" request, as READ misses or additional prefetching may change the segment contents. The most difficult scenario involves disks that discard requested sectors after they are transferred to the host (see section 5.7.1). If the subsequent prefetching activity halts before filling the segment, the extraction algorithm may underestimate the size of the cache segments. For such disks, an attempt must first be made to maximize the prefetching activity by changing the appropriate Mode Page parameters (see table 2).

Some disks dynamically modify the number (and size) of cache segments based on recent request patterns. It may be difficult to accurately characterize the on-board caching behavior of such disks.

\subsubsection{Track-based vs. sector-based}

Some on-board caches allocate space (i.e., segments) based on track size. This has obvious implications for disks with zoned recording: assuming that the cache segments are the size of the largest track, cache space will be underutilized when sectors from smaller tracks are stored in the cache. Track-based caches can be detected by issuing large read requests, allowing prefetch activity to subside, and then determining the contents of the cache, in a manner similar to the extraction of cache segment size (see section 5.7.2). If the range of cached sectors always starts on a track boundary, the odds are fairly high that the cache is track-based. Other potential indicators of track-based caching are prefetching algorithms that stop on track boundaries and segment sizes that appear to be an exact multiple of the current zone's track size.

\subsubsection{Read-on-arrival and write-on-arrival}

Track-based and aggressive sector-based caches can process disk sectors in the order they pass under the read/write head rather than in strictly ascending LBN order. Write-on-arrival can be detected by writing a 1-sector request to the first logical block of a track followed by a track-sized write request starting at the same logical block. If the disk does not have write-on-arrival capability, the request should take approximately two rotations to complete: one rotation to reach the starting block location and one rotation to write the data. With write-on-arrival capability, 
the request should be serviced in much less than two rotations, as it can begin writing sectors to the disk media as soon as they are transferred to the on-board cache. Note that this assumes a path transfer rate that is faster than the media access rate. Choosing a logical track near the spindle will increase the path:media transfer ratio. If the path transfer rate is still too slow, alternate test vectors can be devised. For example, smaller write requests can be used to reduce the possibility of additional rotational delays caused by emptying the on-board cache segment.

Read-on-arrival may apply specifically to requested sectors or to non-requested sectors or to both. To test for this capability, issue a 1-sector read request to sector $\mathrm{N}$, the last logical sector on a specific track. Immediately issue a 1-sector read request to sector $\mathrm{N}-1$. Repeat this sequence several times selecting different values for $\mathrm{N}$. If the disk allows read-on-arrival for non-requested sectors, the second request of each pair should almost always hit in the cache. Read-on-arrival for requested sectors is detected by issuing a 1-sector read request followed by a full-track read request to another track on the same cylinder as the first request. The two requests should be physically aligned (i.e., they should start at the same rotational position). Upon decoding the second request, the disk activates the appropriate $\mathrm{read} /$ write head and performs any necessary actuator fine-tuning. Without read-on-arrival capability, the disk then waits for the first target sector to rotate all the way around to the read/write head. A disk using read-on-arrival, on the other hand, immediately begins reading requested sectors after the head switch is complete. Given knowledge of the path transfer rate, the media transfer rate, and the disconnection behavior (e.g, by preventing disconnection via the Disconnect Privilege Bit of the Identify Message), the rotational latency incurred can be deduced, and thus read-on-arrival capability can be detected.

\subsubsection{Segment allocation}

There are many issues regarding the allocation of cache segments. Segments may or may not be partitioned between READ data and WRITE data (see section 5.7.2). Read requests may or may not be able to hit on data placed in the cache by write requests. When it becomes necessary to assign a "new" segment, the origin of the data in each existing segment (READ, WRITE, or prefetch) may be considered, or a simple allocation policy such as LRU may be employed. When a write request includes sectors that are already present in the on-board cache, the disk must make sure that "old" sectors are invalidated. The timing and extent of such invalidation varies. For example, the entire cache can be invalidated on every WRITE, or just segments with overlapping sectors can be invalided, or just the overlapping sectors can be invalidated.

For track-based caches, a pair of track-sized segments may be assigned to each request, allowing a "ping-pong" behavior where the media access uses one segment (track) while the bus access uses the other. This may cause significant confusion during extraction, especially in conjunction with track-based prefetching algorithms, unless the extraction methodology specifically tests and accounts for this scenario.

Although these issues are complex, each segment allocation characteristic may be tested for using the hypothesisand-test methodology. In each case, the extraction program or user makes an assumption (e.g., that READs can hit on WRITE data) and sets up the cache to allow detection of a read hit or miss (e.g., a test vector with a write request followed by a read request for the same data). 


\subsubsection{Prefetch strategies}

Table 2 includes several prefetch parameters obtainable from the Mode Pages. For example, the amount of prefetched data may be linked to the size of the READ request that preceded the prefetching activity, as is the case when the Multiplication Factor Bit on the Caching Mode Page is set to one. Other prefetch characteristics can only be empirically determined, such as whether or not prefetching activity can traverse track or cylinder boundaries and what happens when the cache segment fills up during prefetch. By issuing different sequences of requests and testing the resulting contents of the cache (i.e., hypothesis and test), various prefetch strategies can be detected.

\subsubsection{Buffer ratios}

The Buffer Full Ratio and Buffer Empty Ratio values specified on the Disconnect-Reconnect Mode Page are required for comprehensive disk modeling. They indicate how full a read cache segment should be and how empty a write cache segment should be before the disk attempts reconnection to the host. However, some disks utilize adaptive algorithms when these values are set to 0x00 [HP93, HP94]. To determine if this is the case, set the Buffer Full Ratio to 0x00 and compute MTBRC(1-sector write, segment-sized read) for the outermost zone. Repeat the extraction with Buffer Full Ratio set to 0x01. If the 0x00 MTBRC is significantly different from the 0x01 MTBRC (i.e., the $0 \mathrm{x} 00$ case is attempting to utilize bus resources more efficiently), an adaptive algorithm may be in use.

\subsection{Path transfer rate}

The data transfer rate between a disk and main memory is a function of the sustainable bandwidth of each component in the I/O data path (e.g., main memory, controllers, buses, adapters, and disk). A single value for the path transfer rate can be determined by comparing service times for two different-sized READ requests that hit in the on-board disk cache. Since the media is not accessed for a READ hit, the only difference between the service times is the additional path transfer time for the larger request.

\section{Model validation}

To validate the described extraction methodology, parameter values were extracted from four different disk drives (see table 1). A detailed disk simulator was configured with the extracted values to allow comparison between measured and modeled disk behavior. In each case, the mean service time reported by the simulator was within $1 \%$ of the value observed for the actual disk (servicing the same workload).

Greater insight can be achieved by comparing the service time distributions for the disk simulator and the actual disk [Ruemmler94]. Figures 4-7 show distributions of measured and simulated service times for a synthetic validation workload of ten thousand requests with the following characteristics:

- $50 \%$ READ requests, $50 \%$ wRITE requests

- $30 \%$ logically sequential requests, $30 \%$ local requests (normal distribution centered on last request, 10000 sector variance), $40 \%$ random requests (uniform distribution) 


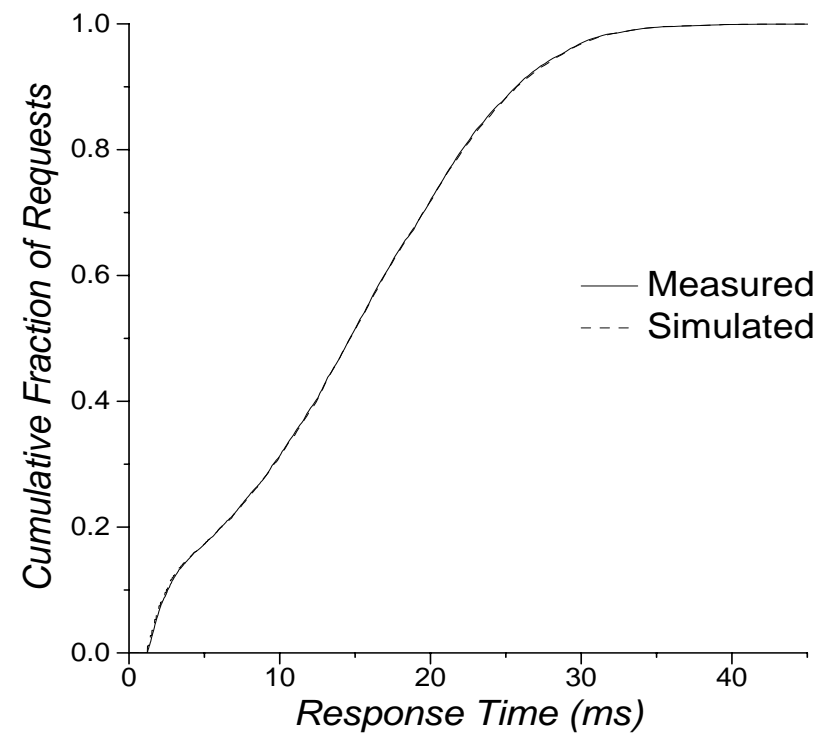

Figure 4: Seagate ST41601N: Service time distributions.

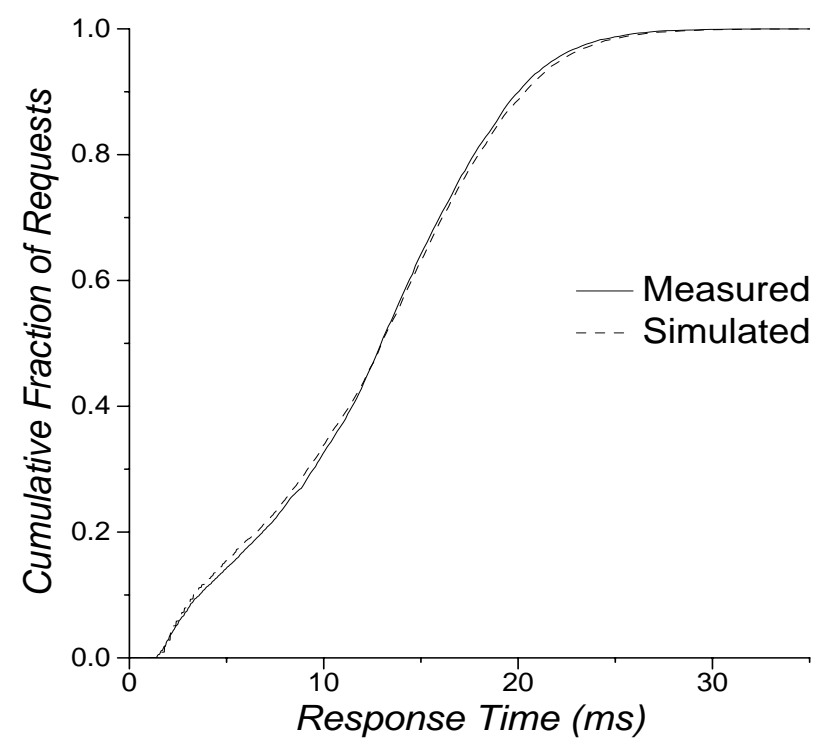

Figure 6: HP C2490A: Service time distributions.

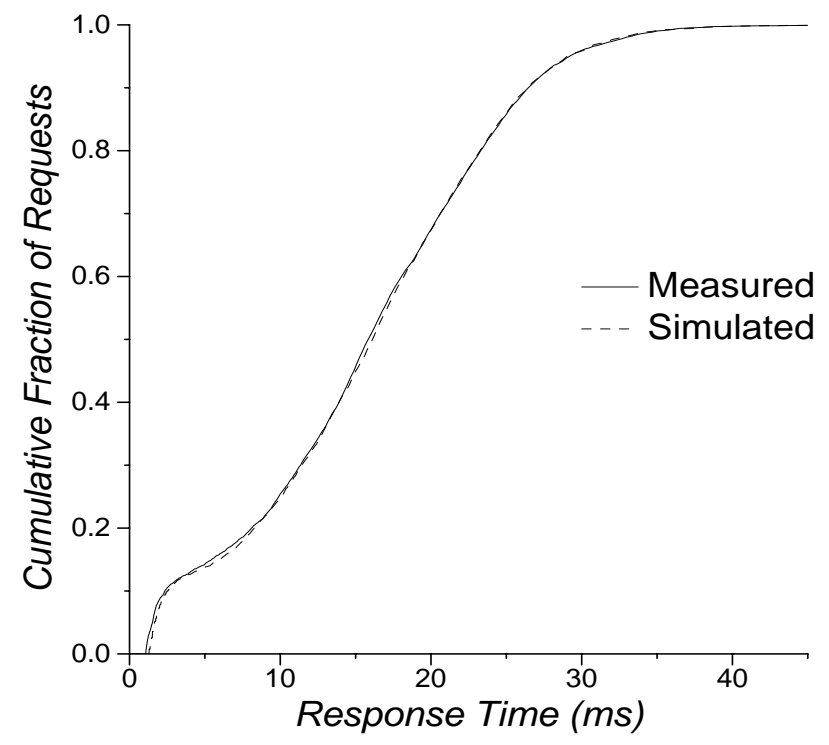

Figure 5: DEC RZ26: Service time distributions.

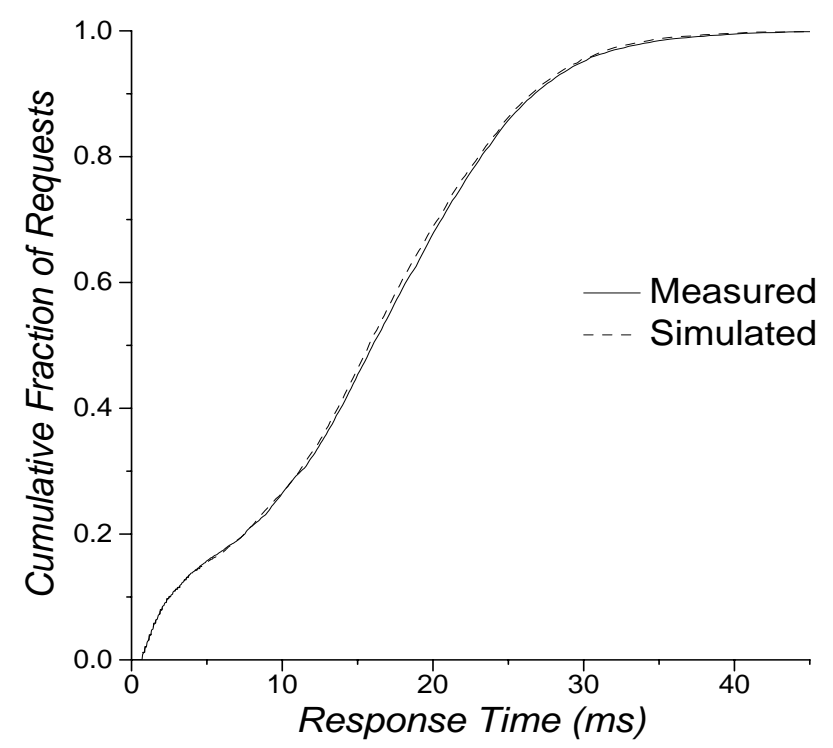

Figure 7: HP C3323A: Service time distributions. 
- $8 \mathrm{~KB}$ mean request size (exponential distribution)

- 0-22 ms request interarrival time (uniform distribution)

Ruemmler and Wilkes defined the root mean square horizontal distance between the two distribution curves as a demerit figure for disk model calibration. The demerit figures for the Seagate ST41601N, DEC RZ26, HP C2490A, and HP C3323A disks are $0.075 \mathrm{~ms}$ ( $0.5 \%$ of the corresponding mean service time), $0.19 \mathrm{~ms}$ ( $1.2 \%$ of the corresponding mean $)^{5}, 0.26 \mathrm{~ms}$ ( $2.0 \%$ of the corresponding mean), and $0.31 \mathrm{~ms}$ ( $1.9 \%$ of the corresponding mean), respectively. These values approach those for the most accurate models discussed by Ruemmler and Wilkes.

While the distribution curves show that the extraction process provides an accurate picture of disk activity over a large number of requests, the mechanical nature of disk drives makes it infeasible to predict individual request service times with a high degree of accuracy. Since a distribution is a smoothing function, the distribution curves hide some of the deviations between measured and simulated activity for the four disks. Figures 8-11 show the corresponding sets of service time density curves for the same validation workloads. A service time granularity of 0.5 ms was chosen to maintain readability while magnifying the differences. Although there are many minor deviations visible, the curve shapes for the simulated disk activity track well with the curve shapes for the actual disk activity.

The close match between the actual disks and the simulator configured with extracted parameters provides strong support for the accuracy of both the model and the extraction process. Also, the generality of the extraction techniques is supported by their success with these different disk drives.

\section{$7 \quad$ Use of extracted data in disk request schedulers}

Disk request service times are often dominated by positioning delays (i.e., seek times and rotational latencies). Schedulers can dynamically reorder queues of pending disk requests to reduce such mechanical delays. Some scheduling algorithms sort disk requests based on their starting LBNs, thus requiring little or no disk-specific knowledge. Such algorithms depend on a strong correlation between the logical "distance" separating two disk requests and the physical distance separating the cylinders containing the two requests' data [Worthington94]. These simple scheduling algorithms attempt to reduce mean seek times by reducing mean seek distances.

More aggressive algorithms, especially those that reduce combined seek and rotational latencies, require accurate knowledge of data layouts, seek curves, head switch times, write settling delays, command processing overheads, and the current rotational position ${ }^{6}$. In particular, a scheduler may need to know how soon a disk can perform a given media access after receiving the appropriate command. This can be difficult to predict due to overlaps between command processing, media transfer, and bus transfer. The inter-request distances measured for MTBRCs provide an additional source of information for aggressive disk request scheduling.

The inter-request distance for MTBRC(X,Y) is a distance along the direction of rotation from the first sector of a request of type $\mathrm{X}$ to the first sector of a request of type $\mathrm{Y}$. At this distance, the second request will incur less than a sector of rotational latency if initiated immediately after the first request. If the distance between a request

\footnotetext{
${ }^{5}$ While validating against the DEC disk, excessive service times were observed for a few requests (28 out of 10,000), probably due to some infrequent disk firmware activity (e.g., thermal recalibration). If these requests are included in the demerit computation, the demerit figure is $0.67 \mathrm{~ms}$ ( $4.3 \%$ of the corresponding mean service time).

${ }^{6}$ SCSI disks often include a spindle synchronization signal that allows rotational position tracking via specialized hardware support.
} 


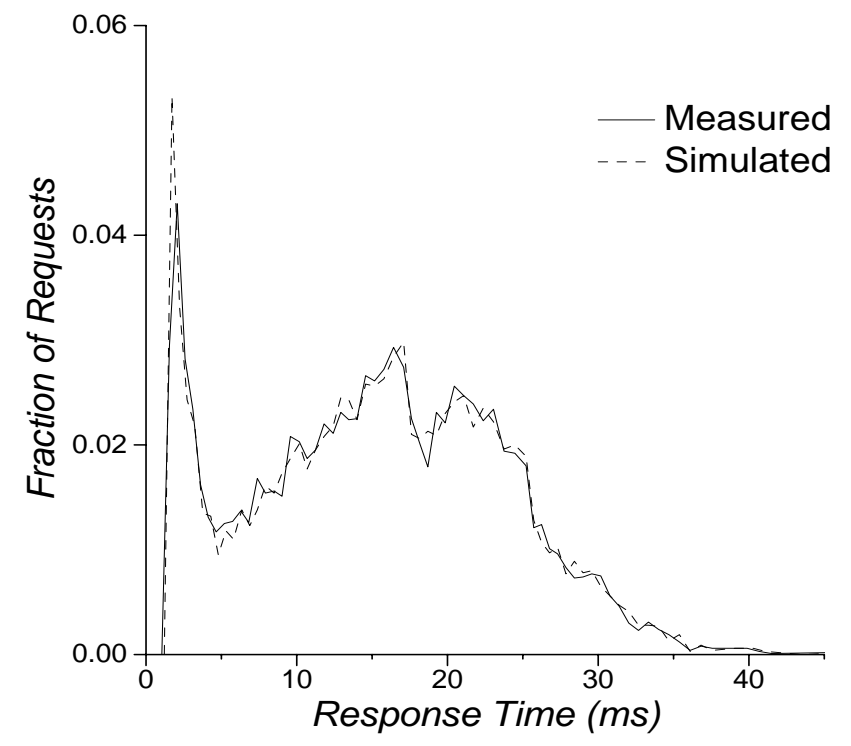

Figure 8: Seagate ST41601N: Service time densities.

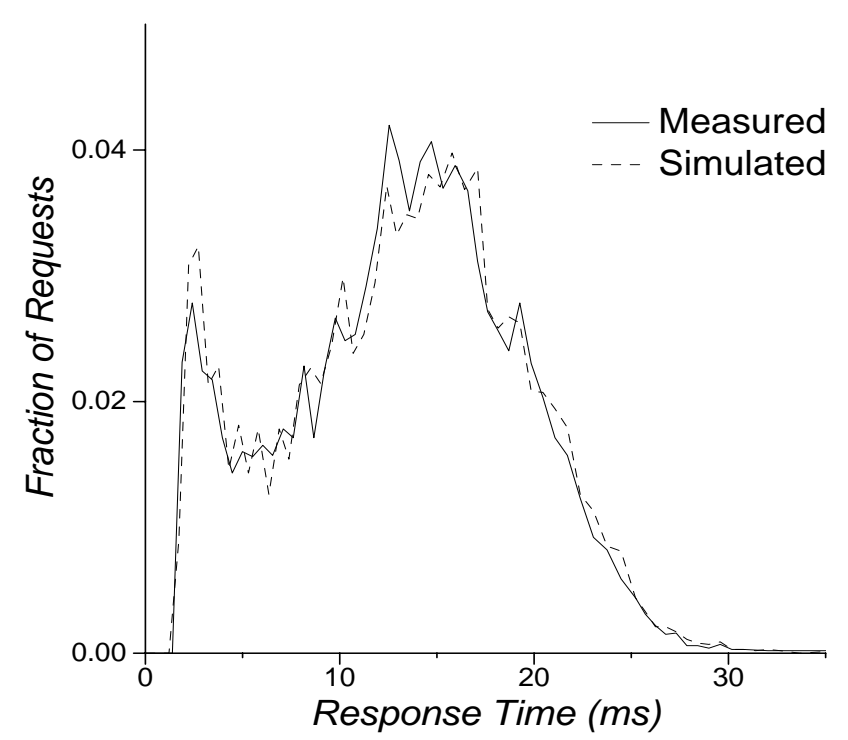

Figure 10: HP C2490A: Service time densities.

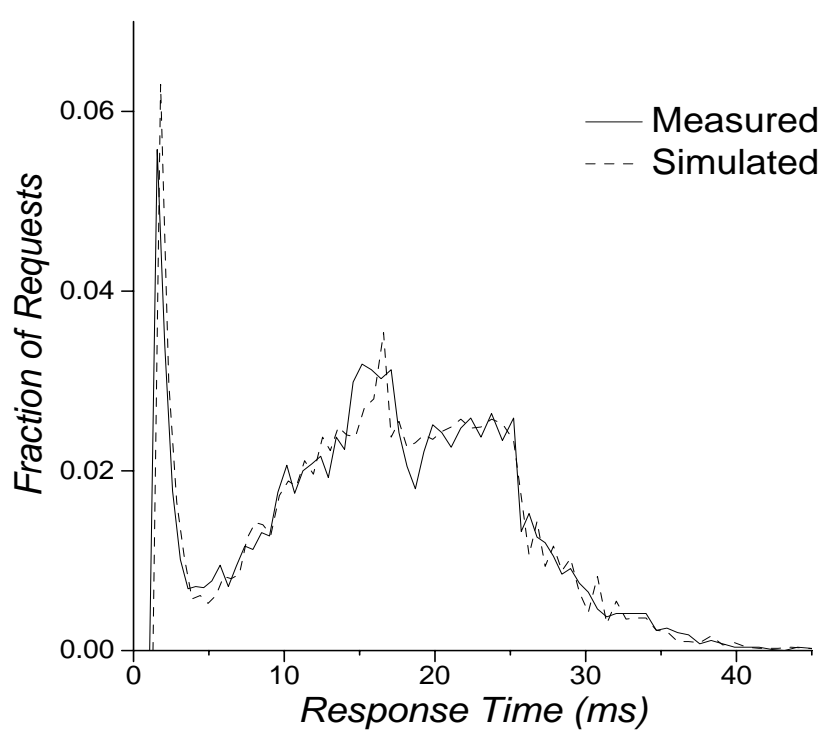

Figure 9: DEC RZ26: Service time densities.

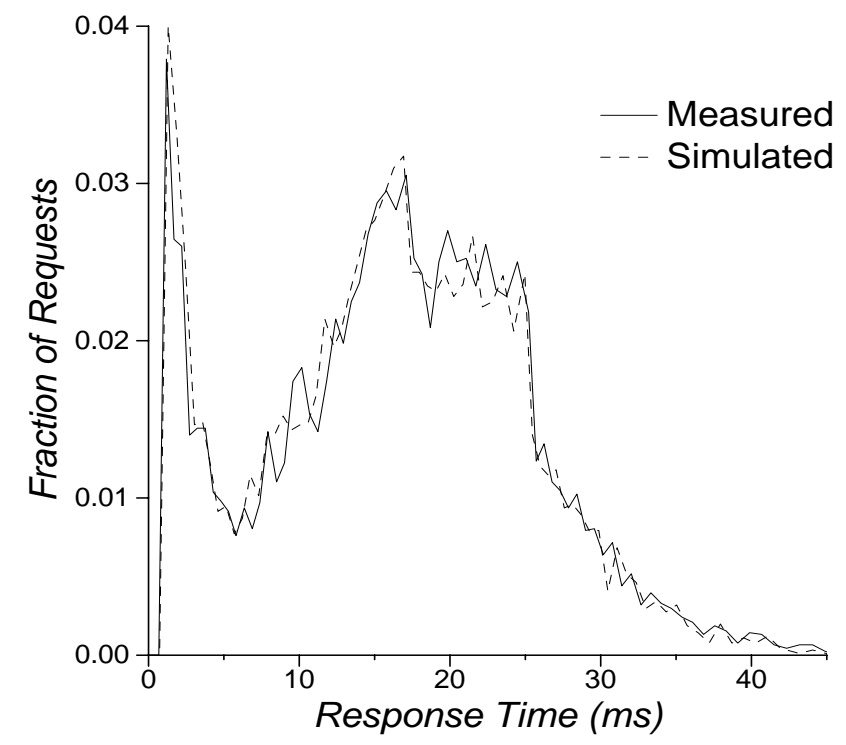

Figure 11: HP C3323A: Service time densities. 
of type $\mathrm{X}$ and a request of type $\mathrm{Y}$ is greater than the extracted inter-request distance for MTBRC(X, Y), the second request will incur correspondingly greater rotational latency. If the distance between the requests is smaller than the inter-request distance, the second request will incur a rotational "miss"; the disk will not be able to interpret the command and perform any necessary repositioning before the first target sector passes the read/write head.

If MTBRC extraction is performed using requests of a common size, such as the file system or database block size, a scheduler can use inter-request distances to predict positioning delays for pending requests. Each time a request of the specified size is serviced, the scheduler may obtain a reference point for future scheduling computations. Due to fluctuations in rotational velocity, the accuracy of a reference point degrades over time. Thus it is necessary to extract MTBRC values using a request size that is representative of the disk's normal workload, so that reference points can be re-established frequently. For example, if the file system block size is used during MTBRC extraction, each single-block request generated by the file system provides an opportunity to generate a more current reference point, provided that the request entails actual media access rather than a read hit in the on-board cache.

The computations necessary to predict positioning delays are best explained with an example:

1. Assume there are 100 sectors per track and the most recent reference point is an N-sector wRITE starting at physical sector 25 that completed 1.10 rotations ago. This reference point is equivalent to an N-sector wRITE starting at physical sector 35 (1.10 rotations beyond sector 25) that has just completed.

2. The inter-request distance previously computed for MTBRC(N-sector wRITE, 1-sector READ on the same track) is 0.20 rotations. A READ request to sector 55 ( 0.20 rotations beyond sector 35$)$ would therefore incur no seek delay, no write settling delay, no head switch delay, and less than one sector of rotational latency.

3. There are two READs in the pending queue, one starting at sector 85 and the other at sector 40 . The first request would incur 0.30 rotations of latency (the distance between sectors 55 and 85 along the direction of rotation). The second would incur 0.85 rotations of latency (the distance between sectors 55 and 40 along the direction of rotation). Therefore, the first request should be scheduled first to minimize mechanical delays.

If a pending request is not on the same track as the current reference point, additional computations are necessary to account for the appropriate seek, head switch, and/or write settling delays. If a scheduler has access to extracted cache parameters, it can give precedence to requests that will hit in the cache and further reduce mean service times [Worthington94].

\section{Conclusions}

Aggressive disk scheduling and accurate performance modeling of disk subsystems both require thorough, quantified understanding of disk drive behavior. This report documents a set of general techniques for efficiently gathering the necessary information from modern SCSI disk drives. Interrogative extraction algorithms request parameter values directly from the disk. Empirical extraction algorithms deduce performance characteristics from measured service times for specific sequences of disk requests. Both methods are needed to obtain the requisite data. The accuracy of the extraction methodology has been demonstrated using a detailed disk simulator configured with extracted parameter values. 
There are a few limitations of the techniques presented:

- The data layout extraction techniques rely on the TRANSLATE ADDRESS form of the SEND/RECEIVE DIAGNOSTIC commands to obtain full logical-to-physical data mappings. For drives lacking this functionality, accurate mapping information must be extracted using alternative means, such as the READ CAPACITY command and the Notch Mode Page.

- The empirical extraction techniques rely on host-observed request service times. Because of this "black-box" methodology, empirical extraction has two major limitations: (1) It is often impossible to distinguish disk performance characteristics from those of other components along the I/O path. (2) With timestamps only taken at the start and end of request service, there is no way of determining when SCSI disconnect/reconnect activity occurs. Other means, such as additional software trace-points or a SCSI bus analyzer, are needed to acquire bus utilization characteristics.

- Modern SCSI drives typically support command queueing at the disk. That is, the disk controller itself can hold multiple pending commands and choose the order in which they are serviced. Test vectors to accurately determine the characteristics of on-board scheduling activity are likely to be lengthy and difficult to design. Because commands are interpreted as soon as the disk receives them, but may be executed at some later point, the extraction of command processing overheads is also complicated by the addition of command queueing.

The difficulties encountered during the course of this study suggest that disk drive manufacturers should be encouraged to provide greater and more accurate information about their products. By allowing other hardware and software developers to better understand and exploit the capabilities of particular disk drive implementations, increased disclosure is more likely than not to lead to a competitive advantage. The easiest way to accomplish this goal is to implement more ANSI-standard Mode Pages, such as the Notch Mode Page, and to increase the set of standardized Mode Pages to include more information on data layout, mechanical delays, cache configuration, command processing delays, and command queueing.

Nevertheless, the extraction methodology documented in this report should provide a good base upon which to develop more comprehensive extraction techniques. The process of manually extracting a full set of parameters for a disk drive was reduced to a matter of hours by the end of the study. An automated extraction program could extract the same set of parameters in a matter of minutes. 


\section{References}

[Clegg86] F. Clegg, G. Ho, S. Kusmer, J. Sontag, "The HP-UX operating system on HP Precision Architecture computers", Hewlett-Packard Journal, Vol. 37, No. 12, December 1986, pp. 4-22.

[HP93] Hewlett-Packard Company, "HP C2490A 3.5-inch SCSI-2 Disk Drives, Technical Reference Manual", Part Number 5961-4359, Boise, ID, Edition 3, September 1993.

[HP94] Hewlett-Packard Company, "HP C3323A 3.5-inch SCSI-2 Disk Drives, Technical Reference Manual”, Part Number 5962-6452, Boise, ID, Edition 2, April 1994.

[Jacobson91] D. Jacobson, J. Wilkes, "Disk scheduling algorithms based on rotational position", Hewlett-Packard Technical Report, HPL-CSP-91-7, Palo Alto, CA, February 26, 1991.

[Ruemmler94] C. Ruemmler, J. Wilkes, “An Introduction to Disk Drive Modeling”, IEEE Computer, Vol. 27, No. 3, March 1994, pp. 17-28.

[SCSI93] “Small Computer System Interface-2", ANSI X3T9.2, Draft Revision 10k, March 17, 1993.

[Seagate92] Seagate Technology, Inc., "SCSI Interface Specification, Small Computer System Interface (SCSI), Elite Product Family", Document \#64721702, Revision D, March 1992.

[Seagate92a] Seagate Technology, Inc., "Seagate Product Specification, ST41600N and ST41601N Elite Disc Drive, SCSI Interface”, Document \#64403103, Revision G, October 1992.

[Seltzer90] M. Seltzer, P. Chen, J. Ousterhout, "Disk Scheduling Revisited", Winter USENIX Proceedings, Washington, D.C., January 1990, pp. 313-324.

[Worthington94] B. Worthington, G. Ganger, Y. Patt, "Scheduling Algorithms for Modern Disk Drives", SIGMETRICS, Nashville, KY, May 1994, pp. 241-251. [An extended version was published as: "Scheduling for Modern Disk Drives and Non-Random Workloads", University of Michigan, Technical Report CSE-TR-194-94, Ann Arbor, MI, March 1994.] 


\section{A Extraction algorithms}

\section{A.1 Computing MTBRC values}

50th and 95th percentile MTBRC(X,Y) values can be obtained in the following manner:

1. Select 3 defect-free cylinders from each of the first, middle, and last zones (for a total of nine cylinders) to be potential targets for the $\mathrm{X}$ requests. If the $\mathrm{X}$ and $\mathrm{Y}$ requests are to be on different cylinders, the corresponding cylinders for the $\mathrm{Y}$ requests must also be defect-free. Perform steps 2 through 8 for each set of 3 cylinders.

By selecting cylinders from the innermost, outermost, and central zones, the final MTBRC values will reflect how mechanical latencies vary as the disk actuator angle changes. The total number of cylinders selected, as with other algorithm parameters discussed below, represents a trade-off between extraction accuracy and efficiency. In general, the parameter values selected make it possible to extract MTBRC values in a matter of seconds.

2. Begin with a short (e.g., 1 sector) inter-request distance (see figure 2). Perform the following steps using slowly increasing inter-request distances until the 50th and 95th percentile values have been determined. To improve extraction efficiency, adjust the inter-request distance by 5-sector increments during the "coarse" tuning phase and 1-sector increments during the subsequent "fine" tuning phase.

3. Pick 20 random targets from the 3 defect-free cylinders selected for the $\mathrm{X}$ requests. If the $\mathrm{X}$ requests are to be READs, pick targets near the logical "beginning" of a track to prevent unwanted head switch or seek activity (see section 5.2). Compute 20 appropriate $\mathrm{Y}$ request targets from the $20 \mathrm{X}$ request targets, the current inter-request distance and any specified track switch or cylinder seek values for the $\mathrm{Y}$ requests.

4. Perform 10 repetitions for each $\mathrm{X}, \mathrm{Y}$ request pair (20 target pairs $\times 10$ repetitions $=200$ pairs total). To improve extraction efficiency, reduce the number of repetitions during the "coarse" tuning phase. If either of the requests are READs, intersperse the requests such that the chances of a hit in the on-board cache are minimized or re-initialize the cache between each 20 pair repetition. That is, make sure that any two repetitions of a given X,Y pair are separated by enough requests so that all corresponding target data has been flushed from the cache. Some knowledge or assumptions about the cache replacement algorithm and the number of cache segments are necessary to satisfy this constraint. The individual completion time differences for all 200 pairs should be measured.

5. Count and discard completion time differences wherein the Y request suffered a rotational "miss". Compute the percentage of requests that were not discarded (i.e., those that did not suffer a rotational miss).

In this context, a rotational miss occurs when a disk does not have enough time to interpret a request and perform any necessary repositioning before the first target sector "passes by" the actuator. Thus requests that incur a rotational miss exhibit request response times containing a significant amount of rotational latency. A miss cutoff value for response times must be chosen that is somewhat greater than the maximum time necessary to complete the second request after the completion of the first, assuming a small quantity of rotational latency. It must also be somewhat less than the time necessary to perform a full rotation, since cleanup and completion, host, and protocol delays occur between the two requests each pair. This makes it possible for a request that incurs a rotational miss to have a response time that is less than a full rotation. For the four disks tested, a miss cutoff value of $3 / 4$ of a rotation was chosen; a rotational miss was recorded if the $\mathrm{Y}$ request service time exceeded $3 / 4$ of a rotation. 
6. Compute the mean time between the completion of the $\mathrm{X}$ requests and the start of the $\mathrm{Y}$ requests (the mean host delay) for the request pairs not discarded in step 5.

7. As the inter-request distance increases, there will come a point where more than $50 \%$ of the $\mathrm{Y}$ requests do not suffer a rotational miss. The 50th percentile MTBRC for the current zone is taken as the mean of the non-discarded request completion differences at this inter-request distance.

8. As the inter-request distance continues to increase, there will come a point where more than $95 \%$ of the $\mathrm{Y}$ requests do not suffer a rotational miss. The 95th percentile MTBRC for the current zone is taken as the mean of the non-discarded request completion differences at this inter-request distance.

9. Combine the information gathered from all three zones by computing the mean of the 50th percentile values and maximum of the 95th percentile values. The inter-request distances (measured in fractions of a rotation) and mean host delays at which the $50 \%$ and $95 \%$ thresholds were crossed should also be noted.

Additional steps can be taken to detect and remove excessive noise. For example, if the three per-zone 50th percentile values computed in step 7 are not within $5-15 \%$ of each other, return to step 3 and repeat the process with a new set of physical request pairs. A filter can also be inserted between steps 4 and 5 that ignores request pairs with completion differences greater than two full rotations ${ }^{7}$ or excessive host delay values. Also, the algorithm implementation should minimize any host delay between the requests in each pair (e.g., reduce the code executed by the host between request pairs), as excessive host delay will artificially increase MTBRC values and inter-request distances.

Using the above algorithm, the 50th and 95th percentile MTBRC values for a specific pair of request types can be determined in a few minutes. By reducing the number of physical request pairs chosen in step 3 and/or the number of repetitions performed in step 4, the extraction cycle can be shortened (at the expense of reduced accuracy).

\section{A.2 Command processing and completion overheads}

As discussed in section 5.6, command processing and completion overheads can only be measured as combined quantities unless the extraction process has access to timing information for when the actual data transfers begin and end. Overhead values can be approximated by solving multiple linear equations populated with extractable values, such as 1-sector media access rates (computed from the data layout and rotation speed), 1-sector path transfer rates (see section 5.8), and MTBRC values (with corresponding mean host delays and inter-request distances).

The six command processing overheads and two completion overheads required for the disk simulator used in this study can be obtained by solving the following set of linear equations:

MTBRC(1-sector write, 1-sector read on the same track) =

Host Delay + Read Miss After Write + Media Xfer + Bus Xfer + Read Completion

Inter-request distance (in time units) $=$

Write Completion + Host Delay + Read Miss After Write + Media Xfer

MTBRC(1-sector write, 1-sector write on same track) =

Host Delay + Write After Write + Bus Xfer + Media Xfer + Write Completion

\footnotetext{
${ }^{7}$ The authors do not understand why a double (or even triple) rotational miss can occur, but the phenomenon occurred several times during the course of the study.
} 
Inter-request distance (in time units) $=$

Write Completion + Host Delay + Write After Write + Bus Xfer + Media Xfer

MTBRC(1-sector read, 1-sector write on same track) =

Host Delay + Write After Read + Bus Xfer + Media Xfer + Write Completion

Inter-request distance (in time units) $=$

Bus Xfer + Read Completion + Host Delay + Write After Read + Bus Xfer + Media Xfer

MTBRC(1-sector read, 1-sector read (miss) on same track) =

Host Delay + Read Miss After Read + Media Xfer + Bus Xfer + Read Completion

Inter-request distance (in time units) =

Bus Xfer + Read Completion + Host Delay + Read Miss After Read + Media Xfer

MTBRC(1-sector read, 1-sector read hit) =

Host Delay + Read Hit After Read + Bus Xfer + Read Completion

MTBRC(1-sector write, 1-sector read hit) =

Host Delay + Read Hit After Write + Bus Xfer + Read Completion

As some of the equations provide potentially conflicting values for a given overhead (e.g., equations A.3 and A.4), a mean value can be used or an equation can be elided. Also, it is necessary to remove one of the unknowns in order to solve the set of equations, as only seven equations remain after resolving conflicts. In the case of the four disks tested, the smaller of the two completion overheads was arbitrarily set to zero. The smaller of the two can be identified from equations A.1 and A.2 or A.5 and A.6. 


\section{B Extracted parameters for specific disk models}

\section{B.1 Seagate ST41601N}

The following measurements were extracted using a system configuration that provided a disk-to-host bandwidth of approximately $5.30 \mathrm{MB} / \mathrm{s}$.

\begin{tabular}{|c|c|}
\hline Parameter & Value \\
\hline Formatted Capacity & $1.37 \mathrm{~GB}$ \\
RPM & $5400(5397$ measured $)$ \\
Diameter & $5^{1 / 4^{\prime \prime}}$ \\
Height & $3.25^{\prime \prime}$ \\
Data Surfaces & 17 \\
Cylinders & 2098 \\
Sector & 512 bytes \\
\hline
\end{tabular}

Table 4: Basic disk drive parameters.

\begin{tabular}{|c|cccccccc|}
\hline Zone & $\begin{array}{c}\text { First } \\
\text { Cylinder }\end{array}$ & $\begin{array}{c}\text { Last } \\
\text { Cylinder }\end{array}$ & $\begin{array}{c}\text { Sectors } \\
\text { Per Track }\end{array}$ & $\begin{array}{c}\text { First Logical } \\
\text { Sector }\end{array}$ & $\begin{array}{c}\text { Track } \\
\text { Skew }\end{array}$ & $\begin{array}{c}\text { Cylinder } \\
\text { Skew }\end{array}$ & $\begin{array}{c}\text { Reserved } \\
\text { Tracks }\end{array}$ & $\begin{array}{c}\text { Spare } \\
\text { Tracks }\end{array}$ \\
\hline 0 & 0 & 625 & 85 & 0 & 3 & 23 & 0 & 0 \\
1 & 626 & 700 & 83 & 46 & 3 & 23 & 0 & 0 \\
2 & 701 & 800 & 82 & 77 & 3 & 22 & 0 & 0 \\
3 & 801 & 925 & 79 & 23 & 3 & 22 & 0 & 0 \\
4 & 926 & 1050 & 78 & 54 & 3 & 21 & 0 & 0 \\
5 & 1051 & 1175 & 76 & 36 & 3 & 21 & 0 & 0 \\
6 & 1176 & 1300 & 73 & 20 & 3 & 20 & 0 & 0 \\
7 & 1301 & 1400 & 72 & 28 & 3 & 20 & 0 & 0 \\
8 & 1401 & 1500 & 69 & 64 & 3 & 19 & 0 & 0 \\
9 & 1501 & 1600 & 68 & 48 & 3 & 19 & 0 & 0 \\
10 & 1601 & 1800 & 65 & 28 & 3 & 18 & 0 & 0 \\
11 & 1801 & 1900 & 63 & 49 & 3 & 18 & 0 & 0 \\
12 & 1901 & 2000 & 62 & 2 & 3 & 17 & 0 & 0 \\
13 & 2001 & 2100 & 61 & 48 & 3 & 17 & 34 & 17 \\
\hline
\end{tabular}

Table 5: Zone specifications. First Logical Sector denotes the physical sector number of the smallest LBN in the zone. Skew values give the additional rotational shift (in sectors) between sequential LBNs across track or cylinder boundaries. Reserved Tracks are used for disk management purposes (e.g., error or defect logs). Spare Tracks are used for remapping LBNs when defective media is detected. 


\begin{tabular}{|c|c|}
\hline $\begin{array}{c}\text { Slipping } \\
\text { Sparing }\end{array}$ & $\begin{array}{c}\text { Sector-based } \\
\text { Sector-based }\end{array}$ \\
\hline Spare Regions & $\begin{array}{c}6 \text { sectors at the logical end of each cylinder } \\
1 \text { cylinder at the logical end of the disk }\end{array}$ \\
\hline
\end{tabular}

Table 6: Defect management.

\begin{tabular}{|c|c|}
\hline $\begin{array}{c}\text { Number of Segments } \\
\text { Segment Size }\end{array}$ & 1 \\
\hline Read-on-arrival & $192 \mathrm{~KB}$ \\
Write-on-arrival & No \\
\hline Requested Data & Discarded before READ completion \\
READ hits on wrITE data & Allowed \\
\hline Prefetch & Obeys Caching Mode Page parameters \\
& Default: 192 KB \\
\hline
\end{tabular}

Table 7: On-board cache characteristics.

\begin{tabular}{|l|c|}
\hline \multicolumn{1}{|c|}{ Overhead } & Value \\
\hline \hline Read Hit After Read & $0.90 \mathrm{~ms}$ \\
Read Hit After Write & $0.50 \mathrm{~ms}$ \\
Read Miss After Read & $1.44 \mathrm{~ms}$ \\
Read Miss After Write & $1.44 \mathrm{~ms}$ \\
Write After Read & $2.01 \mathrm{~ms}$ \\
Write After Write & $1.47 \mathrm{~ms}$ \\
\hline Read Completion & $0.24 \mathrm{~ms}$ \\
Write Completion & $0.00 \mathrm{~ms}$ \\
\hline Head Switch & $0.00 \mathrm{~ms}$ \\
Write Settle & $-0.03 \mathrm{~ms}$ \\
\hline
\end{tabular}

Table 8: 50th percentile command processing, completion, and mechanical overheads. 


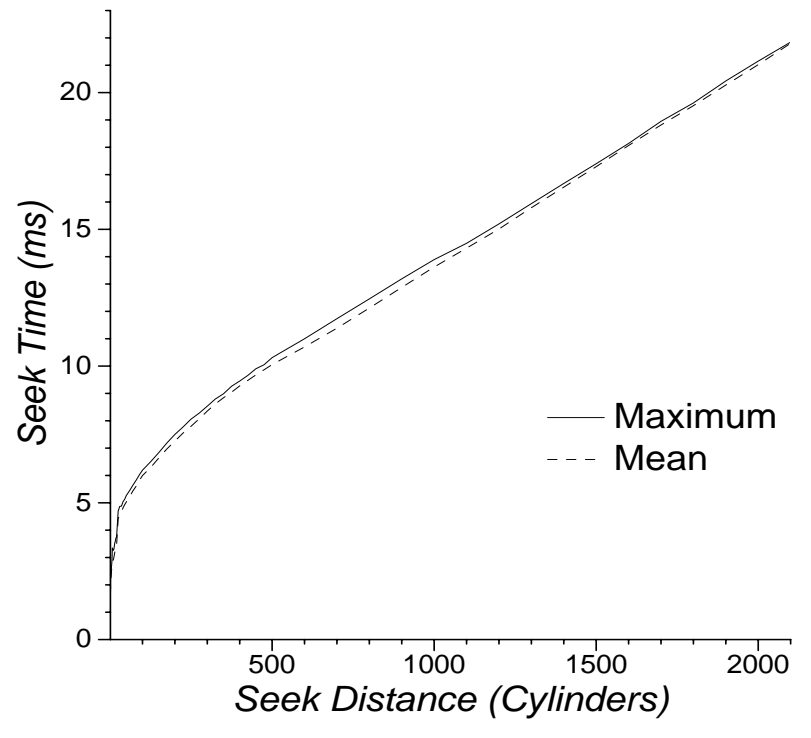

(a) Full seek curve

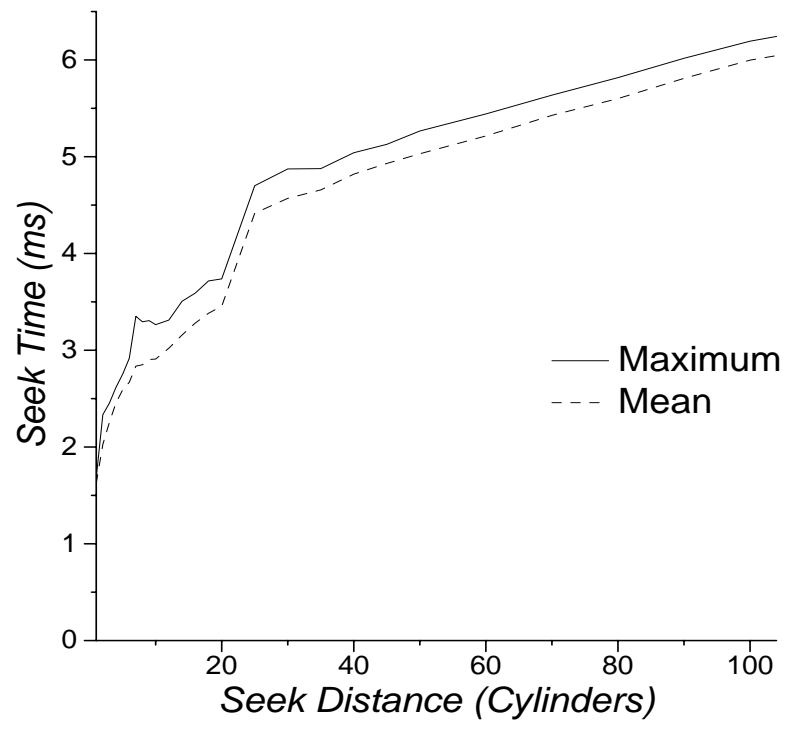

(b) Expanded view of short seeks

Figure 12: Extracted seek curves. 


\section{B.2 DEC RZ26}

The following measurements were extracted using a system configuration that provided a disk-to-host bandwidth of approximately $7.24 \mathrm{MB} / \mathrm{s}$.

\begin{tabular}{|c|c|}
\hline Parameter & Value \\
\hline Formatted Capacity & $1.03 \mathrm{~GB}$ \\
RPM & $5400(5360$ measured $)$ \\
Diameter & $1 \mathrm{r}^{\prime \prime}$ \\
Height & $1.63^{\prime \prime}$ \\
Data Surfaces & 14 \\
Cylinders & 2599 \\
Reserved Cylinders & $0-28($ outermost $)$ \\
Sectors/Track & 58 \\
Sector & 512 bytes \\
Cylinder Skew & 27 \\
Track Skew & 9 \\
\hline
\end{tabular}

Table 9: Basic disk drive parameters. Skew values give the additional rotational shift (in sectors) between sequential LBNs across track or cylinder boundaries. Reserved Cylinders are used for disk management purposes (e.g., error or defect logs).

\begin{tabular}{|c|c|}
\hline $\begin{array}{c}\text { Slipping } \\
\text { Sparing }\end{array}$ & $\begin{array}{c}\text { None } \\
\text { Sector-based }\end{array}$ \\
\hline Spare Regions & 1 sector at the logical end of each track \\
\hline
\end{tabular}

Table 10: Defect management.

\begin{tabular}{|c|c|}
\hline $\begin{array}{l}\text { Number of Segments } \\
\text { Segment Size }\end{array}$ & $\begin{array}{c}10 \text { (track-based instead of request-based) } \\
28.5 \mathrm{~KB} \text { (1 track) }\end{array}$ \\
\hline $\begin{array}{l}\text { Read-on-arrival } \\
\text { Write-on-arrival }\end{array}$ & $\begin{array}{l}\text { Yes } \\
\text { No }\end{array}$ \\
\hline $\begin{array}{c}\text { Requested Data } \\
\text { READ hits on wrITE data }\end{array}$ & $\begin{array}{l}\text { Can be pushed out by prefetch } \\
\text { Only if requests are identical }\end{array}$ \\
\hline Prefetch & $\begin{array}{l}\text { When idle, prefetch to fill segment(s). If } \\
\text { track } \mathrm{N}-1 \text { is present and track } \mathrm{N} \text { is accessed, } \\
\text { prefetch track } \mathrm{N}+1 \text { to replace } \mathrm{N}-1 \text { in cache. }\end{array}$ \\
\hline
\end{tabular}

Table 11: On-board cache characteristics. 


\begin{tabular}{|l|c|}
\hline \multicolumn{1}{|c|}{ Overhead } & Value \\
\hline \hline Read Hit After Read & $1.25 \mathrm{~ms}$ \\
Read Hit After Write & $1.14 \mathrm{~ms}$ \\
Read Miss After Read & $1.55 \mathrm{~ms}$ \\
Read Miss After Write & $1.55 \mathrm{~ms}$ \\
Write After Read & $2.32 \mathrm{~ms}$ \\
Write After Write & $2.08 \mathrm{~ms}$ \\
\hline Read Completion & $0.00 \mathrm{~ms}$ \\
Write Completion & $0.04 \mathrm{~ms}$ \\
\hline Head Switch & $0.70 \mathrm{~ms}$ \\
Write Settle & $-0.45 \mathrm{~ms}$ \\
\hline
\end{tabular}

Table 12: 50th percentile command processing, completion, and mechanical overheads.

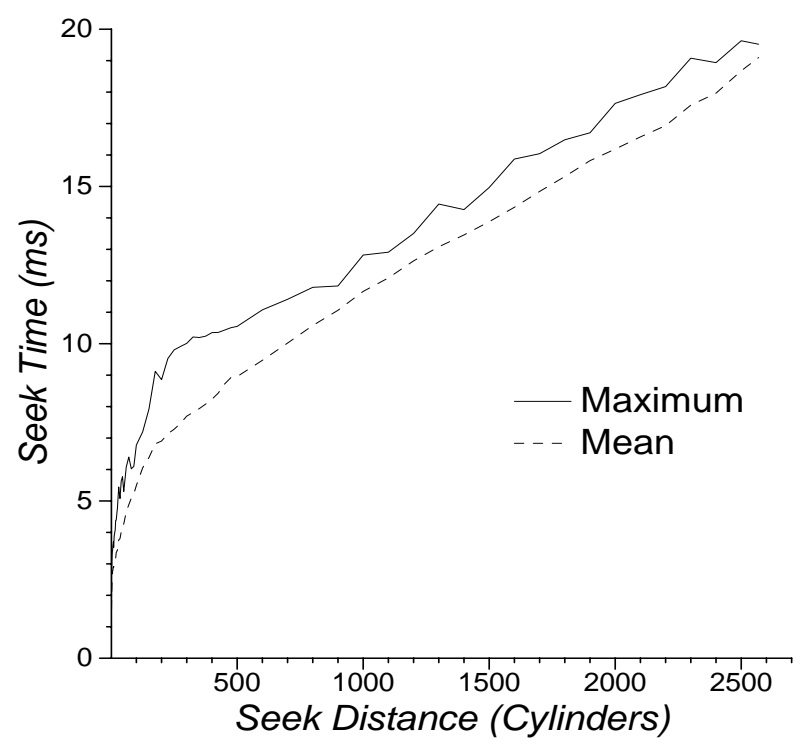

(a) Full seek curve

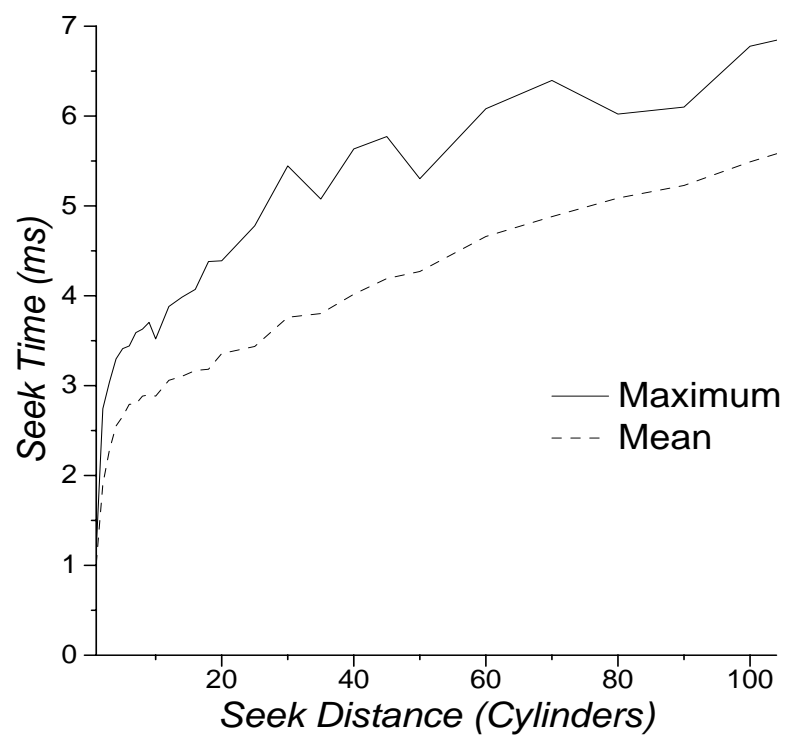

(b) Expanded view of short seeks

Figure 13: Extracted seek curves. 


\section{B.3 HP C2490A}

The following measurements were extracted using a system configuration that provided a disk-to-host bandwidth of approximately $2.67 \mathrm{MB} / \mathrm{s}$.

\begin{tabular}{|c|c|}
\hline Parameter & Value \\
\hline Formatted Capacity & $2.13 \mathrm{~GB}$ \\
RPM & $6400(6398$ measured $)$ \\
Diameter & $3^{1} /^{\prime \prime}$ \\
Height & $1.63^{\prime \prime}$ \\
Data Surfaces & 18 \\
Cylinders & 2630 \\
Sector & 512 bytes \\
\hline
\end{tabular}

Table 13: Basic disk drive parameters.

\begin{tabular}{|c|cccccccc|}
\hline Zone & $\begin{array}{c}\text { First } \\
\text { Cylinder }\end{array}$ & $\begin{array}{c}\text { Last } \\
\text { Cylinder }\end{array}$ & $\begin{array}{c}\text { Sectors } \\
\text { Per Track }\end{array}$ & $\begin{array}{c}\text { First Logical } \\
\text { Sector }\end{array}$ & $\begin{array}{c}\text { Track } \\
\text { Skew }\end{array}$ & $\begin{array}{c}\text { Cylinder } \\
\text { Skew }\end{array}$ & $\begin{array}{c}\text { Reserved } \\
\text { Tracks }\end{array}$ & $\begin{array}{c}\text { Spare } \\
\text { Tracks }\end{array}$ \\
\hline 0 & 0 & 186 & 108 & 90 & 37 & 19 & 22 & 198 \\
1 & 187 & 501 & 104 & 60 & 36 & 18 & 0 & 90 \\
2 & 502 & 785 & 100 & 10 & 34 & 17 & 0 & 72 \\
3 & 786 & 1097 & 96 & 0 & 33 & 17 & 0 & 72 \\
4 & 1098 & 1356 & 92 & 32 & 32 & 16 & 0 & 54 \\
5 & 1357 & 1609 & 88 & 73 & 31 & 16 & 0 & 54 \\
6 & 1610 & 1850 & 84 & 28 & 29 & 15 & 0 & 80 \\
7 & 1851 & 2070 & 80 & 8 & 28 & 14 & 0 & 36 \\
8 & 2071 & 2277 & 76 & 57 & 27 & 14 & 0 & 54 \\
9 & 2278 & 2497 & 72 & 22 & 26 & 13 & 0 & 36 \\
10 & 2498 & 2629 & 68 & 62 & 24 & 13 & 0 & 72 \\
\hline
\end{tabular}

Table 14: Zone specifications. First Logical Sector denotes the physical sector number of the smallest LBN in the zone. Skew values give the additional rotational shift (in sectors) between sequential LBNs across track or cylinder boundaries. Reserved Tracks are used for disk management purposes (e.g., error or defect logs). Spare Tracks are used for remapping LBNs when defective media is detected. 


\begin{tabular}{|c|c|}
\hline $\begin{array}{c}\text { Slipping } \\
\text { Sparing }\end{array}$ & $\begin{array}{c}\text { Track-based } \\
\text { Track-based }\end{array}$ \\
\hline Spare Regions & $\begin{array}{r}\text { Innermost cylinders in each zone } \\
\text { (see table 14) }\end{array}$ \\
\hline
\end{tabular}

Table 15: Defect management.

\begin{tabular}{|c|c|}
\hline $\begin{array}{c}\text { Number of Segments } \\
\text { Segment Size }\end{array}$ & 2 \\
\hline Read-on-arrival & No \\
Write-on-arrival & No \\
\hline Requested Data & Can be pushed out by prefetch \\
Flush cache after write & Yes \\
\hline Prefetch & Obeys Caching Mode Page parameters \\
& Default: 64 KB \\
\hline
\end{tabular}

Table 16: On-board cache characteristics.

\begin{tabular}{|l|c|}
\hline \multicolumn{1}{|c|}{ Overhead } & Value \\
\hline \hline Read Hit After Read & $1.42 \mathrm{~ms}$ \\
Read Hit After Write & N A \\
Read Miss After Read & $2.29 \mathrm{~ms}$ \\
Read Miss After Write & $2.29 \mathrm{~ms}$ \\
Write After Read & $3.22 \mathrm{~ms}$ \\
Write After Write & $2.82 \mathrm{~ms}$ \\
\hline Read Completion & $0.10 \mathrm{~ms}$ \\
Write Completion & $0.00 \mathrm{~ms}$ \\
\hline Head Switch & $1.00 \mathrm{~ms}$ \\
Write Settle & $-0.63 \mathrm{~ms}$ \\
\hline
\end{tabular}

Table 17: 50th percentile command processing, completion, and mechanical overheads. 


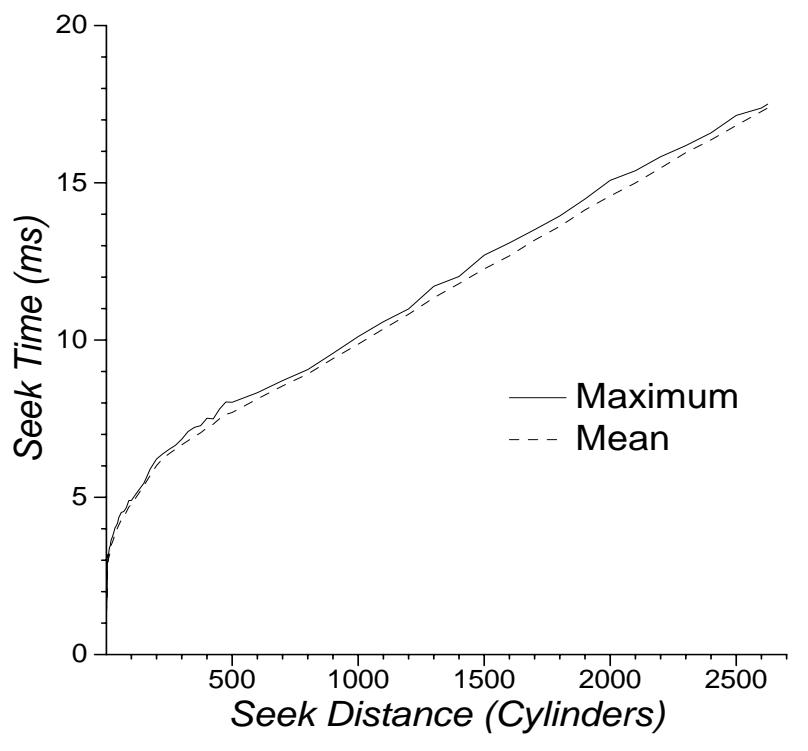

(a) Full seek curve

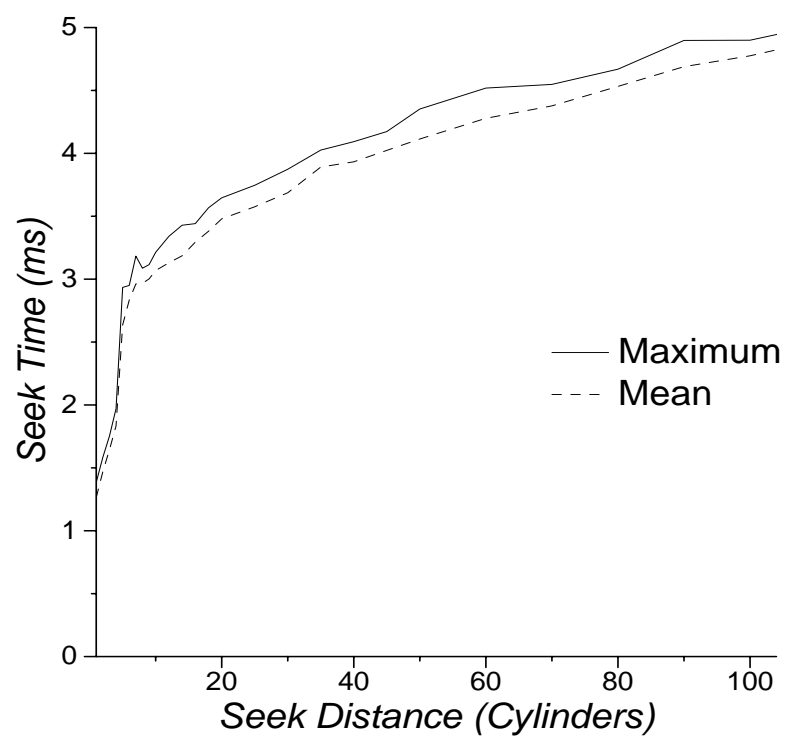

(b) Expanded view of short seeks

Figure 14: Extracted seek curves. 


\section{B.4 HP C3323A}

The following measurements were extracted using a system configuration that provided a disk-to-host bandwidth of approximately $3.54 \mathrm{MB} / \mathrm{s}$.

\begin{tabular}{|c|c|}
\hline Parameter & Value \\
\hline Formatted Capacity & $1.05 \mathrm{~GB}$ \\
RPM & $5400(5396$ measured $)$ \\
Diameter & $3{ }^{\prime \prime}{ }^{\prime \prime}$ \\
Height & $1.00^{\prime \prime}$ \\
Data Surfaces & 7 \\
Cylinders & 2982 \\
Sector & 512 bytes \\
\hline
\end{tabular}

Table 18: Basic disk drive parameters.

\begin{tabular}{|c|cccccccc|}
\hline Zone & $\begin{array}{c}\text { First } \\
\text { Cylinder }\end{array}$ & $\begin{array}{c}\text { Last } \\
\text { Cylinder }\end{array}$ & $\begin{array}{c}\text { Sectors } \\
\text { Per Track }\end{array}$ & $\begin{array}{c}\text { First Logical } \\
\text { Sector }\end{array}$ & $\begin{array}{c}\text { Track } \\
\text { Skew }\end{array}$ & $\begin{array}{c}\text { Cylinder } \\
\text { Skew }\end{array}$ & $\begin{array}{c}\text { Reserved } \\
\text { Tracks }\end{array}$ & $\begin{array}{c}\text { Spare } \\
\text { Tracks }\end{array}$ \\
\hline 0 & 0 & 993 & 120 & 73 & 17 & 34 & 11 & 224 \\
1 & 994 & 1134 & 116 & 48 & 16 & 33 & 0 & 35 \\
2 & 1135 & 1310 & 112 & 23 & 16 & 32 & 0 & 28 \\
3 & 1311 & 1625 & 104 & 47 & 15 & 30 & 0 & 49 \\
4 & 1626 & 1966 & 96 & 48 & 14 & 27 & 0 & 49 \\
5 & 1967 & 2255 & 88 & 72 & 13 & 25 & 0 & 35 \\
6 & 2256 & 2608 & 80 & 48 & 12 & 23 & 0 & 35 \\
7 & 2609 & 2981 & 72 & 64 & 11 & 21 & 0 & 35 \\
\hline
\end{tabular}

Table 19: Zone specifications. First Logical Sector denotes the physical sector number of the smallest LBN in the zone. Skew values give the additional rotational shift (in sectors) between sequential LBNs across track or cylinder boundaries. Reserved Tracks are used for disk management purposes (e.g., error or defect logs). Spare Tracks are used for remapping LBNs when defective media is detected.

\begin{tabular}{|c|c|}
\hline $\begin{array}{c}\text { Slipping } \\
\text { Sparing }\end{array}$ & $\begin{array}{c}\text { Track-based } \\
\text { Track-based }\end{array}$ \\
\hline Spare Regions & $\begin{array}{c}\text { Innermost cylinders in each zone } \\
\text { (see table 19) }\end{array}$ \\
\hline
\end{tabular}

Table 20: Defect management. 


\begin{tabular}{|c|c|}
\hline Number of Segments & 2 \\
Writable Segments & 1 \\
Segment Size & $256 \mathrm{~KB}$ \\
\hline Read-on-arrival & No \\
Write-on-arrival & No \\
\hline Requested Data & Can be pushed out by prefetch \\
READ hits on wrITE data & Allowed \\
\hline \multirow{2}{*}{ Prefetch } & Obeys Caching Mode Page parameters \\
& Default: 64 KB \\
\hline
\end{tabular}

Table 21: On-board cache characteristics.

\begin{tabular}{|l|c|}
\hline \multicolumn{1}{|c|}{ Overhead } & Value \\
\hline \hline Read Hit After Read & $0.35 \mathrm{~ms}$ \\
Read Hit After Write & $1.39 \mathrm{~ms}$ \\
Read Miss After Read & $2.50 \mathrm{~ms}$ \\
Read Miss After Write & $2.50 \mathrm{~ms}$ \\
Write After Read & $3.29 \mathrm{~ms}$ \\
Write After Write & $2.92 \mathrm{~ms}$ \\
\hline Read Completion & $0.24 \mathrm{~ms}$ \\
Write Completion & $0.00 \mathrm{~ms}$ \\
\hline Head Switch & $0.65 \mathrm{~ms}$ \\
Write Settle & $0.07 \mathrm{~ms}$ \\
\hline
\end{tabular}

Table 22: 50th percentile command processing, completion, and mechanical overheads. 


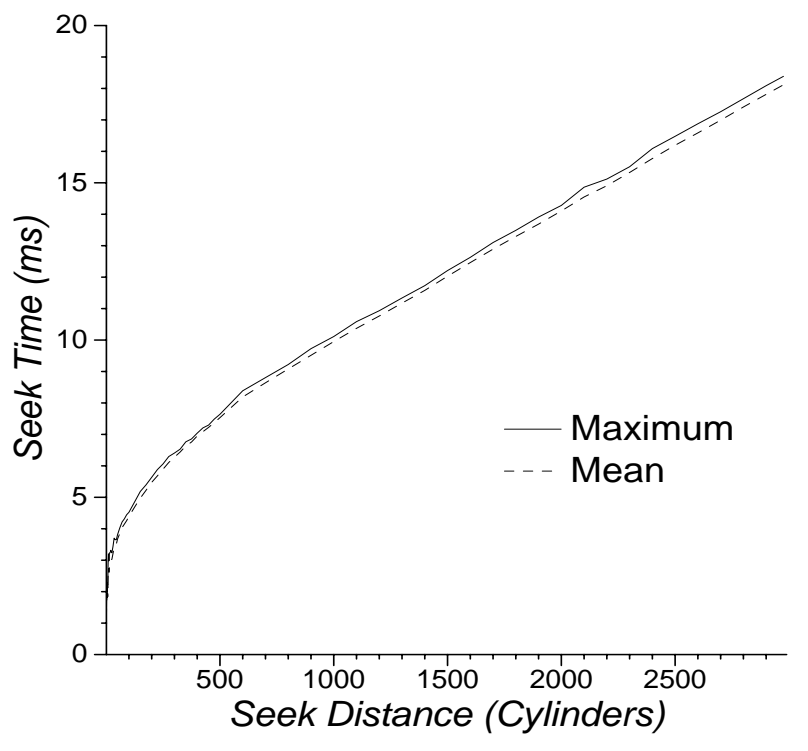

(a) Full seek curve

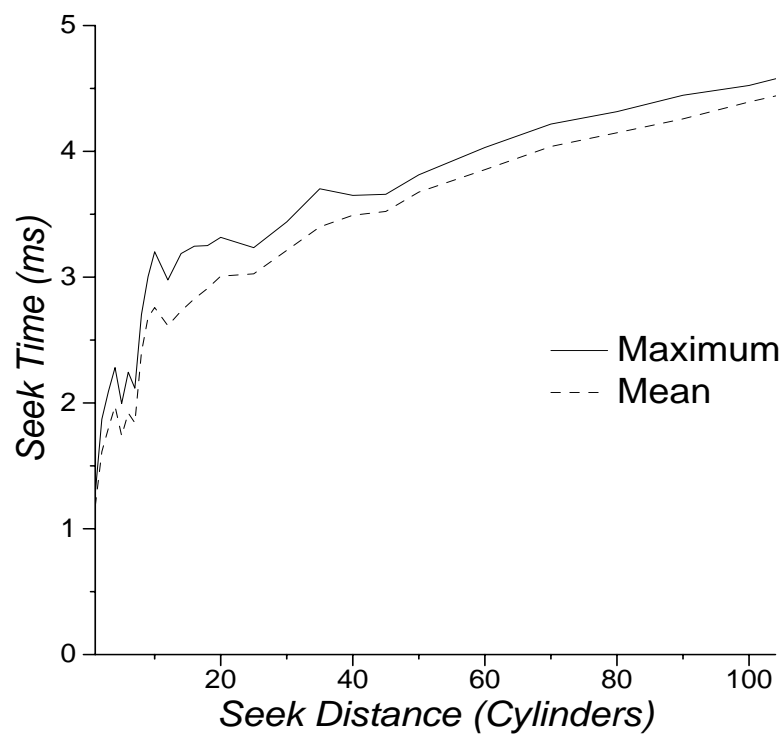

(b) Expanded view of short seeks

Figure 15: Extracted seek curves. 